\title{
FINITE-DIMENSIONALITY OF 2-D MICROPOLAR FLUID FLOW WITH PERIODIC BOUNDARY CONDITIONS
}

Abstract. This paper is devoted to proving the finite-dimensionality of a two-dimensional micropolar fluid flow with periodic boundary conditions. We define the notions of determining modes and nodes and estimate their number. We check how the distribution of the forces and moments through modes influences the estimate of the number of determining modes. We also estimate the dimension of the global attractor. Finally, we compare our results with analogous results for the Navier-Stokes equation.

1. Introduction. There are some heuristic as well as dimensional analysis arguments suggesting that the long-time behavior of a turbulent flow is determined by a finite number of parameters. Therefore the flow has only a finite number of degrees of freedom and can be approximated by a finite system of ordinary differential equations. These arguments are based on a conjecture that rapidly varying, high-wavenumber components decay as fast as they leave lower-wavenumber ingredients unaffected. By Kolmogorov's theory, in 3-dimensional flows only the wavenumbers up to the cutoff value $\lambda_{K}=\left(\varepsilon / \nu^{3}\right)^{1 / 4}$ need to be considered. The question is then reduced, as explained in [17], to finding the number of resolution elements necessary to be considered to describe the behavior of a fluid in a volume, say a cube of side length $l_{0}$. The smallest resolved distance is $l_{d}=1 / \lambda_{K}$ and therefore the number of resolution elements is $\left(l_{0} / l_{d}\right)^{3}$.

A theory of Kraichnan [15], concerning a 2-dimensional turbulent flow, allows us to estimate the number of resolution elements required by $\left(l_{0} / \lambda_{\mathrm{Kr}}\right)^{2}$, where $\lambda_{\mathrm{Kr}}$ is the Kraichnan length $\lambda_{\mathrm{Kr}}=\left(\nu^{3} / \chi\right)^{1 / 6}$ and $\chi$ is the average

2000 Mathematics Subject Classification: 35B40, 35B45, 37L30, 76F20.

Key words and phrases: micropolar fluid, global solution, periodic boundary conditions, determining modes, determining nodes, dimension of global attractor. 
enstrophy dissipation rate. We refer the reader to [3] for a more detailed discussion of turbulent length scales.

The notion of determining modes arises naturally when considering the Fourier decomposition of a flow. There are some results concerning determining modes in the context of 2-dimensional Navier-Stokes equations. Foiaş and Prodi showed in [8] that if a number of Fourier modes of two different solutions have the same asymptotic behavior, then the entire solutions also have the same asymptotic behavior. Subsequent work has been aimed at estimating how many low modes are necessary to determine the behavior of a flow. The most recent results are in [6] for the case of no-slip boundary conditions and in [12] for the case of periodic boundary conditions. The number of determining modes for a 2-D micropolar fluid flow with no-slip boundary conditions was estimated in [23].

In many practical situations, for instance in physical experiments, data are collected from measurements at finitely many points in the domain of the flow. A natural question is how many measurement points are necessary to determine the long-term behavior of the flow. This leads to the notion of determining nodes, introduced by Foiaş and Temam in [9]. The most recent estimate for the lowest number of determining nodes for the Navier-Stokes equation in the space-periodic case was derived in [12].

Another approach to describing the asymptotic behavior of a flow with a finite number of parameters is to use a global attractor. For every trajectory $u$ in the phase space we can choose, by the "Shadowing Lemma" (cf. [21]), a trajectory $u_{A}$ lying on the attractor that is arbitrarily close to $u$ in an interval of time, that is, $\left|u(t)-u_{A}(t)\right|<\varepsilon$ for $t \in\left(t_{0}, t_{1}\right)$. On the other hand, a global attractor has finite Hausdorff and fractal dimension, so we can parametrize it with a finite number of parameters (cf. [7], [10], [19]). Therefore we can describe approximately the long-term behavior of the flow by a finite number of parameters.

There are many results concerning the dimension of an attractor for the Navier-Stokes equation with a variety of boundary conditions, e.g. periodic boundary conditions [26] or a pipe-like domain with arbitrary inflow at infinity [20]. The dimension of an attractor for micropolar fluid equations with various boundary conditions was estimated in [2], [18].

In this paper we will consider all the above mentioned ways of determining the long-time behavior of a micropolar fluid flow by a finite number of parameters. We estimate the lowest number of determining modes (Theorem 2) and nodes (Theorem 3), and the dimension of a global attractor (Theorem 4).

We will consider the micropolar fluid equations, which in the two-dimensional case have the form (cf. [18]) 


$$
\begin{aligned}
& \frac{\partial u}{\partial t}-\left(\nu+\nu_{r}\right) \Delta u+(u \cdot \nabla) u+\nabla p=2 \nu_{r} \operatorname{rot} \omega+f, \\
& \operatorname{div} u=0, \\
& \frac{\partial \omega}{\partial t}-\alpha \Delta \omega+(u \cdot \nabla) \omega+4 \nu_{r} \omega=2 \nu_{r} \operatorname{rot} u+g,
\end{aligned}
$$

where $u=\left(u_{1}, u_{2}\right)$ is the velocity field, $p$ is the pressure and $\omega$ is the microrotation field interpreted as the angular velocity of particles. In the two-dimensional case we assume that the axis of rotation of particles is perpendicular to the $x_{1}, x_{2}$ plane. The fields $f=\left(f_{1}, f_{2}\right)$ and $g$ are the external forces and moments respectively. The positive constants $\nu, \nu_{r}, \alpha$ are the viscosity coefficients, and

$$
\operatorname{rot} u=\frac{\partial u_{2}}{\partial x_{1}}-\frac{\partial u_{1}}{\partial x_{2}}, \quad \operatorname{div} u=\frac{\partial u_{1}}{\partial x_{1}}+\frac{\partial u_{2}}{\partial x_{2}}, \quad \operatorname{rot} \omega=\left(\frac{\partial \omega}{\partial x_{2}},-\frac{\partial \omega}{\partial x_{1}}\right) .
$$

We supplement these equations with the initial conditions

$$
u(x, 0)=u_{0}(x), \quad \omega(x, 0)=\omega_{0}(x)
$$

and periodic boundary conditions

$$
u\left(x+L e_{i}, t\right)=u(x, t), \quad \omega\left(x+L e_{i}, t\right)=\omega(x, t) \quad \forall x \in \mathbb{R}^{2} \forall t>0,
$$

where $e_{1}, e_{2}$ is the usual basis of $\mathbb{R}^{2}$ and $L$ is the period in the $i$ th direction. We give the existence theorem in the next section after defining the relevant function spaces.

This paper is organized as follows: In Section 2 we introduce the function spaces and operators used throughout, and we present the main results. Section 3 contains some a priori estimates. In Sections 4 and 5 we estimate the number of determining modes and determining nodes, respectively. In Section 6 we recall the notions of fractal and Hausdorff dimension and estimate the dimension of a global atractor.

\section{Mathematical setting of the problem and the main results.} In this section we introduce some function spaces, trilinear forms $b$ and $b_{1}$, the Stokes and $-\Delta$ operators and the rot operator.

Function spaces. Set $Q=(0, L)^{2}$. For every Banach space $X$ we will denote by $\mathbb{X}$ the space $X \times X$ with the standard product norm.

$L^{q}$ is the usual Lebesgue space $L^{q}(Q)$ for $q \in[1, \infty]$. We denote the scalar product in $L^{2}$ by $(\cdot, \cdot)$ and the norm in $L^{2}$ by $|\cdot|$ when it does not lead to confusion.

$H^{m}, m \in \mathbb{N}$, are the usual Sobolev spaces $H^{m}(Q)$ of functions whose derivatives up to order $m$ are square integrable, with the norm

$$
\|u\|_{m}=\left(\sum_{|\alpha| \leq m} \int_{Q}\left|D^{\alpha} u\right|^{2} d x\right)^{1 / 2} .
$$


We denote by $H_{\mathrm{per}}^{m}(Q), m \in \mathbb{N}$, the space of real functions in $H_{\mathrm{loc}}^{m}\left(\mathbb{R}^{n}\right)$ which are periodic with period $L$ in each coordinate direction: $u\left(x+L e_{i}\right)=$ $u(x), i=1,2$. It is a Hilbert space with the scalar product

$$
(u, v)_{m}=\sum_{|\alpha| \leq m} \int_{Q} D^{\alpha} u(x) D^{\alpha} v(x) d x
$$

and the induced norm $|\cdot|_{m}$. The functions in $H_{\text {per }}^{m}(Q)$ are explicitly characterized by their Fourier series expansion:

$$
H_{\mathrm{per}}^{m}(Q)=\left\{u=\sum_{k \in \mathbb{Z}^{n}} u_{k} e^{2 i \pi k / L \cdot x}: \bar{u}_{k}=u_{-k},|u|_{m}=\sum_{k \in \mathbb{Z}^{n}}|k|^{2 m}\left|u_{k}\right|^{2}<\infty\right\},
$$

where $k / L=\left(k_{1} / L, k_{2} / L\right)$. The norm $|u|_{m}$ is equivalent to the norm $\left\{\sum_{k \in \mathbb{Z}^{n}}\left(1+|k|^{2 m}\right)\left|u_{k}\right|^{2}\right\}^{1 / 2}$. We also set

$$
\dot{H}_{\mathrm{per}}^{m}(Q)=\left\{u \in H_{\mathrm{per}}^{m}(Q): u_{0}=0\right\} .
$$

We denote by $H$ and $V$ the divergence-free subsets of $\dot{H}_{\text {per }}^{0}(Q)$ and $\dot{\mathbb{H}}_{\text {per }}^{1}(Q)$, respectively. We equip $V$ with the scalar product and the Hilbert norm

$$
((u, v))=\sum_{i=1}^{n}\left(\frac{\partial u}{\partial x_{i}}, \frac{\partial v}{\partial x_{i}}\right), \quad\|u\|=\{((u, u))\}^{1 / 2}
$$

This norm is equivalent to the norm induced by $H_{\text {per }}^{1}(Q)$, and $V$ is a Hilbert space for this scalar product.

One can check that $\dot{H}_{\text {per }}^{-m}$ is the dual space to $\dot{H}_{\text {per }}^{m}$ we also denote the dual space to $V$ as $V^{\prime}$.

Let $\mathcal{H}$ and $\mathcal{V}$ denote $H \times \dot{H}_{\text {per }}^{0}$ and $V \times \dot{H}_{\text {per }}^{1}$, respectively, with standard product norms.

$L^{q}(0, T ; X)$, where $X$ is a Banach space, is the space of strongly measurable functions $u:(0, T) \rightarrow X$ with the following norm:

$$
\|u\|_{L^{q}(0, T ; X)}= \begin{cases}\left(\int_{0}^{T}\|u(t)\|_{X}^{q} d t\right)^{1 / q}, & 1 \leq q<\infty, \\ \operatorname{ess~sup}_{t \in(0, T)}\|u(t)\|_{X}, & q=\infty .\end{cases}
$$

$C([0, T] ; X)$, where $X$ is a Banach space, is the space of continuous functions $u:(0, T) \rightarrow X$ with the usual norm.

Stokes and $-\Delta$ operators. Let us consider the Stokes problem, obtained from the Navier-Stokes equation by neglecting all time-dependent and nonlinear terms, with periodic boundary conditions (1.5): for given $f \in \dot{\mathbb{H}}_{\text {per }}^{0}$ or $\dot{\mathbb{H}}_{\text {per }}^{-1}$, find $u \in \dot{\mathbb{H}}_{\text {per }}^{1}$ and $p \in L^{2}$ such that 


$$
-\Delta u+\nabla p=f, \quad \nabla \cdot u=0 .
$$

It is known (cf. e.g. $[21,25])$ that in this case the Stokes operator $A$ is simply $-\Delta$ (provided that $f \in H$ ) with domain $D(A)=\dot{\mathbb{H}}_{\text {per }}^{2} \cap H$. The operator $A$ is one-to-one from $D(A)$ onto $H$.

The operator $A^{-1}$ is linear and continuous from $H$ into $D(A)$. Since the injection of $D(A)$ in $H$ is compact, we can consider $A^{-1}$ as a compact operator in $H$. It is also self-adjoint as an operator in $H$. Hence it has a sequence of eigenfunctions $w_{j}, j \in \mathbb{N}$, which form an orthonormal basis of $H$,

$$
\begin{aligned}
& A w_{j}=\lambda_{j} w_{j}, \quad w_{j} \in D(A), \\
& 0<\lambda_{1} \leq \lambda_{2} \leq \ldots, \quad \lambda_{j} \rightarrow \infty \quad \text { as } j \rightarrow \infty .
\end{aligned}
$$

Let us consider the Poisson equation with periodic boundary condition, that is: for given $g \in \dot{H}_{\text {per }}^{-1}$ find $\omega \in \dot{H}_{\text {per }}^{1}$ such that

$$
-\Delta \omega=g \text {. }
$$

The operator $-\Delta$ has the same properties as the Stokes operator: it is oneto-one from $D\left(A_{1}\right)=\dot{H}_{\text {per }}^{2} \cap \dot{H}_{\text {per }}^{0}$ onto $\dot{H}_{\text {per. }}^{0}$ The operator $A_{1}^{-1}$ is linear, continuous from $\dot{H}_{\mathrm{per}}^{0}$ onto $D\left(A_{1}\right)$ and compact as an operator in $\dot{H}_{\mathrm{per}}^{0}$. Although the eigenvalues are the same, the eigenfunctions are different, because $\omega$ is a scalar function. We denote the eigenfunctions of $-\Delta$ by $\varrho_{k}$. Let $A_{1}$ be the $-\Delta$ operator with domain $D\left(A_{1}\right)=\dot{H}_{\text {per }}^{2} \cap \dot{H}_{\text {per }}^{0}$.

We can express every $u \in H$ and every $\omega \in \dot{H}_{\text {per }}^{0}$ as

$$
u(x, t)=\sum_{k=1}^{\infty} u_{k}(t) w_{k}(x), \quad \omega(x, t)=\sum_{k=1}^{\infty} \omega_{k}(t) \varrho_{k}(x) .
$$

The Galerkin projectors corresponding to the first $m$ modes are

$$
P_{m} u(x, t)=\sum_{k=1}^{m} u_{k}(t) w_{k}(x), \quad P_{m}^{1} \omega_{i}(x, t)=\sum_{k=1}^{m} \omega_{k}(t) \varrho_{k}(x) .
$$

We also denote the projections onto modes higher than $m$ by $Q_{m}$ and $Q_{m}^{1}$ respectively,

$$
Q_{m} u(x, t)=\sum_{k=m+1}^{\infty} u k(t) w_{k}(x), \quad Q_{m}^{1} \omega(x, t)=\sum_{k=m+1}^{\infty} \omega_{k}(t) \varrho_{k}(x) .
$$

Trilinear forms. We define trilinear forms $b$ and $b_{1}$ as follows:

$$
b(u, v, w)=\sum_{i, j=1}^{2} \int_{Q} u_{i} \frac{\partial v_{j}}{\partial x_{i}} w_{j} d x
$$


for all $u, v, w \in V$, and

$$
b_{1}(u, \omega, \psi)=\sum_{i=1}^{2} \int_{Q} u_{i} \frac{\partial \omega}{\partial x_{i}} \psi d x
$$

for all $u \in V$ and all scalar functions $\omega, \psi \in \dot{H}_{\text {per }}^{1}(Q)$. The forms $b$ and $b_{1}$ are skew-symmetric with respect to the last two coordinates,

$$
b(u, v, w)=-b(u, w, v), \quad b_{1}(u, \omega, \varrho)=-b_{1}(u, \varrho, \omega),
$$

which implies the orthogonality property

$$
b(u, v, v)=0, \quad b_{1}(u, \omega, \omega)=0 .
$$

In the 2-dimensional space-periodic case the form $b$ has one more orthogonality property (cf. [25]):

$$
b(u, u, A u)=0 \quad \forall u \in D(A),
$$

which the form $b_{1}$ does not have-it is not true that $b_{1}\left(u, \omega, A_{1} \omega\right)=0$ for all $u \in D(A)$ and $\omega \in D\left(A_{1}\right)$. The lack of this orthogonality property causes that the a priori estimates we obtain are more involved than analogous estimates for the Navier-Stokes equation with periodic boundary conditions.

We get some estimates of the forms $b$ and $b_{1}$ using the Ladyzhenskaya inequality [16]

$$
\|u\|_{L^{4}} \leq\left(\frac{6}{\pi}\right)^{1 / 4}|u|^{1 / 2}\|u\|^{1 / 2} \quad \text { for all } u \in \dot{H}_{\mathrm{per}}^{1},
$$

and the Agmon inequality $[1,11]$

$$
\|u\|_{L^{\infty}} \leq \frac{1}{\sqrt{\pi}}|u|^{1 / 2}|A u|^{1 / 2} \quad \text { for all } u \in D(A) .
$$

We also use the Hölder inequalities:

$$
\begin{aligned}
&|b(u, v, w)| \leq c_{1}|u|^{1 / 2}\|u\|^{1 / 2}\|v\| \cdot|w|^{1 / 2}\|w\|^{1 / 2}, \quad u, v, w \in V, \\
&|b(u, v, A w)| \leq c_{1}|u|^{1 / 2}\|u\|^{1 / 2}\|v\|^{1 / 2}|A v|^{1 / 2}|A w|, \quad u \in V, v, w \in D(A), \\
&\left|b_{1}(u, \omega, \psi)\right| \leq c_{1}|u|^{1 / 2}\|u\|^{1 / 2}|\psi|^{1 / 2}\|\psi\|^{1 / 2}\|\omega\|, \quad u, \omega, \psi \in \dot{H}_{\mathrm{per}}^{1}, \\
&\left|b_{1}\left(u, \omega, A_{1} \psi\right)\right| \leq c_{1}|u|^{1 / 2}|A u|^{1 / 2}\|\omega\| \cdot\left|A_{1} \psi\right|, \\
& u \in D(A), \omega \in \dot{H}_{\mathrm{per}}^{1}, \psi \in D\left(A_{1}\right), \\
&\left|b_{1}\left(u, \omega, A_{1} \psi\right)\right| \leq c_{1}|u|^{1 / 2}|A u|^{1 / 2}\|\omega\| \cdot\left|A_{1} \psi\right|, \\
& u \in D(A), \omega \in \dot{H}_{\mathrm{per}}^{1}, \psi \in D\left(A_{1}\right),
\end{aligned}
$$

for an appropriate constant $c_{1}$. 
The rot operator has the following properties:

$$
\begin{aligned}
\int_{Q} \operatorname{rot} u \cdot \omega d x & =\int_{Q} \operatorname{rot} \omega \cdot u d x, \quad \int_{Q}|\operatorname{rot} \omega|^{2} d x=\int_{Q}|\nabla \omega|^{2} d x, \\
\int_{Q}|\operatorname{rot} u|^{2} d x & =\int_{Q}|\nabla u|^{2} d x,
\end{aligned}
$$

for all $u \in V$ and $\omega \in \dot{H}_{\text {per. }}^{1}$.

Main results. The existence and uniqueness of solutions for this model as well as existence of a global attractor were proved in [24]. We also assume (as in [24]) that the space averages of $u, \omega, f$ and $g$ vanish.

Theorem 1. Let $f \in L^{2}(0, T ; H), g \in L^{2}\left(0, T ; \dot{H}_{\text {per }}^{0}\right)$ for each $T>0$ and let $u_{0} \in H, \omega_{0} \in \dot{H}_{\mathrm{per}}^{0}$. There exists a unique weak solution of problem (1.1)-(1.3), that is, a pair of functions $(u, \omega)$ where

$$
\begin{array}{ll}
u \in C([0, T] ; H) \cap L^{2}(0, T ; V) & \text { for each } T>0, \\
\omega \in C\left([0, T] ; \dot{H}_{\mathrm{per}}^{0}\right) \cap L^{2}\left(0, T ; \dot{H}_{\mathrm{per}}^{1}\right) & \text { for each } T>0,
\end{array}
$$

such that $u(x, 0)=u_{0}(x), \omega(x, 0)=\omega_{0}(x)$ and satisfying the following identities:

$$
\begin{aligned}
\frac{d}{d t}(u(t), \varphi)+\left(\nu+\nu_{r}\right)(\nabla u(t), \nabla \varphi)+b(u(t), & u(t), \varphi) \\
& =2 \nu_{r}(\operatorname{rot} \omega(t), \varphi)+(f, \varphi)
\end{aligned}
$$

for all $\varphi \in V$, and

$$
\begin{aligned}
\frac{d}{d t}(\omega(t), \psi)+\alpha(\nabla \omega(t), \nabla \psi)+b_{1}(u(t), \omega(t), \psi) & +4 \nu_{r}(\omega(t), \psi) \\
& =2 \nu_{r}(\operatorname{rot} u, \psi)+(g(t), \psi)
\end{aligned}
$$

for all $\psi \in \dot{H}_{\mathrm{per}}^{1}(Q)$, in the sense of scalar distributions on $(0, \infty)$.

In the first two theorems that we prove in this paper, we consider the difference between two solutions of micropolar fluid equations. Let us denote by $\left(u_{1}, \omega_{1}\right)$ and $\left(u_{2}, \omega_{2}\right)$ solutions corresponding to two possibly different pairs of the external forces and moments $\left(f_{1}, g_{1}\right)$ and $\left(f_{2}, g_{2}\right)$ respectively, with the corresponding pressure terms $p=p(x, t)$ and $q=q(x, t)$. We set $u=u_{1}-u_{2}, \omega=\omega_{1}-\omega_{2}, f=f_{1}-f_{2}$ and $g=g_{1}-g_{2}$.

It is assumed that the external forces $f_{1}, f_{2}$ and moments $g_{1}, g_{2}$ have the same asymptotic behavior for large time, that is,

$$
\left\|f_{1}(x, t)-f_{2}(x, t)\right\|_{\mathbb{H}^{-1}}^{2}+\left\|g_{1}(x, t)-g_{2}(x, t)\right\|_{H^{-1}}^{2} \rightarrow 0 \quad \text { as } t \rightarrow \infty
$$

for determining modes and

$$
\left|f_{1}(x, t)-f_{2}(x, t)\right|^{2}+\left|g_{1}(x, t)-g_{2}(x, t)\right|^{2} \rightarrow 0 \quad \text { as } t \rightarrow \infty
$$


for determining nodes. The estimates obtained in this paper will be given in terms of the asymptotic strength of forces and moments measured in their $L^{2}$ and $H^{-1}$ norms, that is,

$$
\begin{aligned}
\widetilde{F} & =\limsup _{t \rightarrow \infty}\left(\left|f_{1}(t)\right|^{2}+\left|g_{1}(t)\right|^{2}\right)^{1 / 2}, \\
\widetilde{F}_{-1} & =\limsup _{t \rightarrow \infty}\left(\left\|f_{1}(t)\right\|_{\mathbb{H}^{-1}}^{2}+\left\|g_{1}(t)\right\|_{H^{-1}}^{2}\right)^{1 / 2} .
\end{aligned}
$$

Let us notice that in the autonomous case, $\widetilde{F}=\left(|f|^{2}+|g|^{2}\right)^{1 / 2}$.

Definition 1. The first $m$ modes associated with $P_{m}$ and $P_{m}^{1}$ are called determining modes if the condition

$$
\int_{Q}\left(\left|P_{m} u_{1}(x, t)-P_{m} u_{2}(x, t)\right|^{2}+\left|P_{m}^{1} \omega_{1}(x, t)-P_{m}^{1} \omega_{2}(x, t)\right|^{2}\right) d x \rightarrow 0
$$

as $t \rightarrow \infty$, together with the condition (2.10) for the forces and moments, implies

$$
\int_{Q}\left(\left|Q_{m} u_{1}(x, t)-Q_{m} u_{2}(x, t)\right|^{2}+\left|Q_{m}^{1} \omega_{1}(x, t)-Q_{m}^{1} \omega_{2}(x, t)\right|^{2}\right) d x \rightarrow 0
$$

as $t \rightarrow \infty$.

An estimate of the number of determining modes is given in the following theorem.

Theorem 2. Let $f_{i} \in L^{2}(0, T ; \mathcal{H}), g_{i} \in L^{2}\left(0, T ; L^{2}\right)$ for $i=1$, 2 . If the forces and moments satisfy condition (2.10), then the first $m$ modes are determining in the sense of Definition 1 provided that

$$
m \geq \frac{16 \nu_{r}^{2}}{d \lambda_{1} \alpha k_{1}}+\frac{8 c_{1}^{2}}{d \lambda_{1} k_{3} k_{1}^{3}} \widetilde{F}_{-1}^{2} .
$$

In order to prove this estimate we derive a differential inequality for $\left|Q_{m} u(t)\right|^{2}+\left|Q_{m}^{1} \omega(t)\right|^{2}$ and then we estimate $m$ by checking the assumptions of the generalized Gronwall lemma (Lemma 2; cf. [5]).

We consider a set $\Sigma=\left\{x^{1}, \ldots, x^{N}\right\}$ of $N$ measurement points (called nodes). We assume that these points are uniformly distributed within the domain $Q$ in the sense that $Q$ may be covered by $N$ identical squares $Q_{1}, \ldots, Q_{N}$ such that exactly one $x^{i}$ is in each square: $x^{i} \in Q_{i}$.

We assume that both flows have the same time-asymptotic behavior at the measurement points. This can be written in the form

$$
\begin{gathered}
\max _{j=1, \ldots, N}\left|u_{1}\left(x^{j}, t\right)-u_{2}\left(x^{j}, t\right)\right| \rightarrow 0 \quad \text { as } t \rightarrow \infty, \\
\max _{j=1, \ldots, N}\left|\omega_{1}\left(x^{j}, t\right)-\omega_{2}\left(x^{j}, t\right)\right| \rightarrow 0 \quad \text { as } t \rightarrow \infty .
\end{gathered}
$$


We want to estimate how many points of observation are necessary to determine the asymptotic behavior of the flow in the following sense:

Definition 2. The set $\Sigma=\left\{x^{1}, \ldots, x^{N}\right\}$ is called a set of determining nodes if (2.14), (2.15) together with the condition (2.11) for the forces and moments implies

$$
\int_{Q}\left(\left|u_{1}(x, t)-u_{2}(x, t)\right|^{2}+\left|\omega_{1}(x, t)-\omega_{2}(x, t)\right|^{2}\right) d x \rightarrow 0 \quad \text { as } t \rightarrow \infty .
$$

THEOREM 3. Let $Q$ be a domain covered by $N$ identical squares $Q_{1}$, $\ldots, Q_{N}$ and consider a set $\Sigma=\left\{x^{1}, \ldots, x^{N}\right\}$ of points in $Q$ distributed one in each square: $x^{i} \in Q_{i}$ for $1 \leq i \leq N$. Let $f_{1}$ and $f_{2}$ be two forcing terms in $L^{2}(0, \infty ; H)$ and $g_{1}$ and $g_{2}$ be two moments in $L^{2}\left(0, \infty ; \dot{H}_{\mathrm{per}}^{0}\right)$, satisfying (2.11). Then $\Sigma$ is a set of determining nodes in the sense of Definition 2 for the 2-dimensional micropolar fluid equations with periodic boundary conditions provided that

$$
\begin{aligned}
N \geq & \frac{c}{\lambda_{1} k_{1}}\left\{\frac{8 \nu_{r}^{2}}{\alpha}-2 \nu_{r}\right. \\
& +\left(\frac{c_{1}^{2} c^{1 / 2}}{\lambda_{1} \nu}+\frac{c_{1}}{\alpha}\right) \cdot\left(\frac{5 \alpha k_{2}+32 \nu_{r}^{2}}{\alpha k_{1}^{2} k_{2}} \widetilde{F}^{2}+\frac{16 C \widehat{c}_{1}}{\alpha \nu k_{1}^{2} k_{2}^{3}} \widetilde{F}^{6} \exp \left(\widehat{c}_{2}+\widehat{c}_{3} \widetilde{F}^{4}\right)\right) \\
& \left.+\frac{16 c_{1}^{4} \widehat{c}_{1}}{\lambda_{1} \alpha \nu k_{1} k_{2}} \widetilde{F}^{4} \exp \left(\widehat{c}_{2}+\widehat{c}_{3} \widetilde{F}^{4}\right)\right\} .
\end{aligned}
$$

To obtain the above estimate we derive a differential inequality for the $H^{1}$-norm of the difference of solutions and bound $N$ from below by checking the assumptions of the generalized Gronwall lemma.

The following theorem concerns the dimension of a global attractor.

THEOREM 4. There exists a constant $C_{0}$ such that if $N$ is the integer satisfying

$$
N-1<2 C_{0}\left(k_{1}^{3} k_{2}\right)^{-1 / 2} \widetilde{F} \leq N,
$$

where $k_{1}, k_{2}$ are as in (3.2) below, then the $N$-dimensional volume element in the phase space $\mathcal{H}$ is exponentially decaying; moreover the Hausdorff dimension of the attractor $\mathcal{A}_{\nu_{r}}, \nu_{r} \geq 0$, is less than or equal to $N$ and its fractal dimension is less than or equal to $2 \mathrm{~N}$.

The dimension of the attractor is estimated by using Lyapunov exponents.

3. A priori estimates. In this section we derive some a priori estimates. Since we will consider the asymptotic behavior of solutions we estimate their norms in terms of the asymptotic strength of forces and moments. To this end we set 


$$
\begin{aligned}
\widetilde{F} & =\limsup _{t \rightarrow \infty}\left(|f(t)|^{2}+|g(t)|^{2}\right)^{1 / 2}, \\
\widetilde{F}_{-1} & =\limsup _{t \rightarrow \infty}\left(\|f(t)\|_{\mathbb{H}^{-1}}^{2}+\|g(t)\|_{H^{-1}}^{2}\right)^{1 / 2},
\end{aligned}
$$

and

$$
k_{1}=\min \{\nu, \alpha\}, \quad k_{2}=k_{1} \lambda_{1} .
$$

Inequalities (3.3), (3.6) and (3.8) below come from [24]. Let us consider the following one:

$$
\frac{d}{d t}\left(|u(t)|^{2}+|\omega(t)|^{2}\right)+k_{2}\left(|u(t)|^{2}+|\omega(t)|^{2}\right) \leq k_{2}^{-1}\left(|f(t)|^{2}+|g(t)|^{2}\right) .
$$

Integrating it with respect to $t$ in the interval $(0, t)$ we obtain

$$
\begin{aligned}
& |u(t)|^{2}+|\omega(t)|^{2} \leq\left|u_{0}\right|^{2}+\left|\omega_{0}\right|^{2}+k_{2}^{-1} \int_{0}^{t}\left(|f(s)|^{2}+|g(s)|^{2}\right) d s \\
& \leq\left|u_{0}\right|^{2}+\left|\omega_{0}\right|^{2}+k_{2}^{-1}\left(\|f\|_{L^{2}(0, \infty ; H)}^{2}+\|g\|_{L^{2}\left(0, \infty ; \dot{H}_{\mathrm{per}}^{0}\right)}^{2}\right),
\end{aligned}
$$

which implies a uniform bound on the norm of the solution in $\mathcal{H}$.

Applying Gronwall's inequality to (3.3) we obtain a bound on the norm of solutions for large times, which is independent of the initial condition. First we have

$$
\begin{aligned}
|u(t)|^{2}+|\omega(t)|^{2} \leq & e^{-k_{2}\left(t-t_{0}\right)}\left(\left|u\left(t_{0}\right)\right|^{2}+\left|\omega\left(t_{0}\right)\right|^{2}\right) \\
& +k_{2}^{-2}\left(1-e^{-k_{2}\left(t-t_{0}\right)}\right)\left(\|f\|_{L^{\infty}\left(t_{0}, t ; H\right)}^{2}+\|g\|_{L^{\infty}\left(t_{0}, t ; \dot{H}_{\mathrm{per}}^{0}\right)}^{2}\right),
\end{aligned}
$$

hence for $t_{0}$ and $t$ large enough,

$$
|u(t)|^{2}+|\omega(t)|^{2} \leq \frac{2}{k_{2}^{2}} \widetilde{F}^{2} .
$$

To estimate the average of the square of the norm of solutions in $\mathcal{V}$ we use the inequality

$$
\begin{aligned}
\frac{d}{d t}\left(|u(t)|^{2}+|\omega(t)|^{2}\right)+k_{1}\left(\|u(t)\|^{2}+\|\omega(t)\|^{2}\right) & \\
& \leq k_{2}^{-1}\left(|f(t)|^{2}+|g(t)|^{2}\right) .
\end{aligned}
$$

By integration we obtain

$$
\begin{aligned}
|u(t+T)|^{2}+\mid \omega(t+ & T)\left.\right|^{2}+k_{1} \int_{t}^{t+T}\left(\|u(s)\|^{2}+\|\omega(s)\|^{2}\right) d s \\
& \leq k_{2}^{-1} \int_{t}^{t+T}\left(|f(s)|^{2}+|g(s)|^{2}\right) d s+|u(t)|^{2}+|\omega(t)|^{2} .
\end{aligned}
$$

Since $|u(t)|^{2}+|\omega(t)|^{2}$ is uniformly bounded with respect to $t$ (cf. (3.4)), for $t$ and $T$ large enough we have 


$$
\begin{aligned}
& \frac{1}{T} \int_{t}^{t+T}\left(\|u(s)\|^{2}+\|\omega(s)\|^{2}\right) d s \\
& \quad \leq\left(k_{1} k_{2}\right)^{-1} \frac{1}{T} \int_{t}^{t+T}\left(|f(s)|^{2}+|g(s)|^{2}\right) d s+\frac{1}{T}\left(|u(t)|^{2}+|\omega(t)|^{2}\right) \\
& \quad \leq \frac{2}{k_{1} k_{2}} \widetilde{F}^{2} .
\end{aligned}
$$

In order to derive two more estimates we consider the inequality

$$
\begin{aligned}
& \quad \frac{d}{d t}\left(\|u(t)\|^{2}+\|\omega(t)\|^{2}\right)+\frac{k_{1}}{2}\left(|A u(t)|^{2}+\left|A_{1} \omega(t)\right|^{2}\right) \\
& \leq\left(\frac{2 C}{\alpha^{2} \nu}|u(t)|^{2}\|\omega(t)\|^{2}+\frac{8 \nu_{r}}{\alpha}\right)\left(\|u(t)\|^{2}+\|\omega(t)\|^{2}\right)+\frac{2}{k_{1}}\left(|f(t)|^{2}+|g(t)|^{2}\right) .
\end{aligned}
$$

Setting

$$
\begin{aligned}
& y(t)=\|u(t)\|^{2}+\|\omega(t)\|^{2}, \quad \widetilde{g}(t)=\left(\frac{2 C}{\alpha^{2} \nu}|u(t)|^{2}\|\omega(t)\|^{2}+\frac{8 \nu_{r}}{\alpha}\right), \\
& \widetilde{h}(t)=\frac{2}{k_{1}}\left(|f(t)|^{2}+|g(t)|^{2}\right),
\end{aligned}
$$

we infer from (3.8) that

$$
\frac{d y}{d t} \leq \widetilde{g} y+\widetilde{h} .
$$

We check the assumptions of the uniform Gronwall lemma (cf. [26]). If $t>t_{0}$ (so that estimates (3.5) and (3.7) hold), for some $r$ we have

$$
\begin{aligned}
& \int_{t}^{t+r} \widetilde{g}(s) d s \leq \frac{8 \nu_{r}^{2} r}{\alpha}+\frac{8 C r}{\alpha^{2} \nu k_{1} k_{2}^{3}} \widetilde{F}^{4} \equiv a_{1}, \quad \int_{t}^{t+r} \widetilde{h}(s) d s \leq \frac{3 r}{k_{1}} \widetilde{F}^{2} \equiv a_{2}, \\
& \int_{t}^{t+r} y(s) d s \leq \frac{2 r}{k_{2}^{2}} \widetilde{F}^{2} \equiv a_{3} .
\end{aligned}
$$

Therefore by uniform Gronwall's lemma we obtain

$$
\|u(t)\|^{2}+\|\omega(t)\|^{2} \leq \frac{2+3 k_{2} r}{k_{1} k_{2}} \widetilde{F}^{2} \exp \left(\frac{8 \nu_{r}^{2} r}{\alpha}+\frac{8 C r}{\alpha^{2} \nu k_{1} k_{2}^{3}} \widetilde{F}^{4}\right)
$$

for all $t>t_{0}+r$. Set

$$
\widehat{c}_{1}=\frac{2+3 k_{2} r}{k_{1} k_{2}}, \quad \widehat{c}_{2}=\frac{8 \nu_{r}^{2} r}{\alpha}, \quad \widehat{c}_{3}=\frac{8 C r}{\alpha^{2} \nu k_{1} k_{2}^{3}} .
$$

Then (3.9) becomes

$$
\|u(t)\|^{2}+\|\omega(t)\|^{2} \leq \widehat{c}_{1} \widetilde{F}^{2} \exp \left(\widehat{c}_{2}+\widehat{c}_{3} \widetilde{F}^{4}\right) .
$$


Now we want to derive an estimate on the average of the square of the norm of solutions in $D(A) \times D\left(A_{1}\right)$. Integrating (3.8) in $(t, t+T)$ we get

$$
\begin{aligned}
\|u(t+T)\|^{2}+ & \|\omega(t+T)\|^{2}-\|u(t)\|^{2}-\|\omega(t)\|^{2} \\
& +\frac{k_{1}}{2} \int_{t}^{t+T}\left(|A u(s)|^{2}+\left|A_{1} \omega(s)\right|^{2}\right) d s \\
\leq & \int_{t}^{t+T}\left\{\left(\frac{2 C}{\alpha^{2} \nu}|u(t)|^{2}\|\omega(t)\|^{2}+\frac{8 \nu_{r}}{\alpha}\right)\left(\|u(t)\|^{2}+\|\omega(t)\|^{2}\right)\right. \\
& \left.+\frac{2}{k_{1}}\left(|f(t)|^{2}+|g(t)|^{2}\right)\right\} d s,
\end{aligned}
$$

hence

$$
\begin{aligned}
& \frac{1}{T} \int_{t}^{t+T}\left(|A u(s)|^{2}+\left|A_{1} \omega(s)\right|^{2}\right) d s \\
& \leq \frac{1}{T} \frac{2}{k_{1}}\left(\|u(t)\|^{2}+\|\omega(t)\|^{2}\right)+\frac{4}{k_{1}^{2}} \frac{1}{T} \int_{t}^{t+T}\left(|f(s)|^{2}+|g(s)|^{2}\right) d s \\
&+\frac{2}{k_{1}} \frac{1}{T} \int_{t}^{t+T}\left(\frac{2 C}{\alpha^{2} \nu}\left(|u(s)|^{2}+|\omega(s)|^{2}\right)\left(\|u(s)\|^{2}+\|\omega(s)\|^{2}\right)+\frac{8 \nu_{r}^{2}}{\alpha}\right) \\
& \cdot\left(\|u(s)\|^{2}+\|\omega(s)\|^{2}\right) d s .
\end{aligned}
$$

Since solutions are uniformly bounded in the $\mathcal{V}$ norm for large $t$, for $t$ and $T$ large enough we obtain

$$
\begin{aligned}
& \frac{1}{T} \int_{t}^{t+T}\left(|A u(s)|^{2}+\left|A_{1} \omega(s)\right|^{2}\right) d s \\
& \leq \frac{5}{k_{1}^{2}} \widetilde{F}^{2}+\frac{16 \nu_{r}^{2}}{\alpha k_{1}} \frac{1}{T} \int_{t}^{t+T}\left(\|u(s)\|^{2}+\|\omega(s)\|^{2}\right) d s \\
& \quad+\frac{4 C}{\alpha^{2} \nu k_{1}} \frac{1}{T} \int_{t}^{t+T}\left(|u(s)|^{2}+|\omega(s)|^{2}\right)\left(\|u(s)\|^{2}+\|\omega(s)\|^{2}\right)^{2} d s \\
& \leq\left(\frac{5}{k_{1}^{2}}+\frac{32 \nu_{r}^{2}}{\alpha k_{1}^{2} k_{2}}\right) \widetilde{F}^{2}+\frac{8 C}{\alpha^{2} \nu k_{1} k_{2}^{2}} \widetilde{F}^{2} \\
& \cdot \frac{1}{T} \int_{t}^{t+T}\left(\|u(s)\|^{2}+\|\omega(s)\|^{2}\right)\left(\|u(s)\|^{2}+\|\omega(s)\|^{2}\right) d s \\
& \leq\left(\frac{5}{k_{1}^{2}}+\frac{32 \nu_{r}^{2}}{\alpha k_{1}^{2} k_{2}}\right) \widetilde{F}^{2}+\frac{16 C \widehat{c}_{1}}{\alpha^{2} \nu k_{1}^{2} k_{2}^{3}} \widetilde{F}^{6} \exp \left(\widehat{c}_{2}+\widehat{c}_{3} \widetilde{F}^{4}\right) .
\end{aligned}
$$


The estimate we derive below is necessary to estimate the number of determining modes in terms of the $H^{-1}$ norm of the forces and moments. Taking the scalar product of (1.1) with $u$ in $H$ we obtain

$$
\frac{1}{2} \frac{d}{d t}|u|^{2}+\left(\nu+\nu_{r}\right)\|u\|^{2}=2 \nu_{r}(\operatorname{rot} \omega, u)+(f, u)
$$

because $b(u, u, u)=0$. We estimate the terms of the RHS of (3.13) as follows:

$$
\begin{aligned}
2 \nu_{r}(\operatorname{rot} \omega, u) & =2 \nu_{r}(\omega, \operatorname{rot} u) \leq 2 \nu_{r}|\omega| \cdot\|u\| \leq 2 \nu_{r}|\omega|^{2}+\frac{\nu_{r}}{2}\|u\|^{2}, \\
(f, u) & \leq\|f\|_{\mathbb{H}^{-1}}\|u\|_{H} \leq \frac{\nu}{2}\|u\|^{2}+\frac{1}{2 \nu}\|f\|_{\mathbb{H}^{-1}}^{2} .
\end{aligned}
$$

We treat (1.3) in an analogous way. We multiply it by $\omega$, integrate over $Q$ and obtain

$$
\frac{1}{2} \frac{d}{d t}|\omega|^{2}+\alpha\|\omega\|^{2}+4 \nu_{r}|\omega|^{2}=2 \nu_{r}(\operatorname{rot} u, \omega)+(g, \omega) .
$$

The terms of the RHS of (3.15) are estimated as

$$
\begin{aligned}
2 \nu_{r}(\operatorname{rot} u, \omega) & \leq 2 \nu_{r}|\omega|^{2}+\frac{\nu_{r}}{2}\|u\|^{2}, \\
(g, \omega) & \leq\|g\|_{H^{-1}}\|\omega\| \leq \frac{\alpha}{2}\|\omega\|^{2}+\frac{1}{2 \alpha}\|g\|_{H^{-1}}^{2} .
\end{aligned}
$$

Adding equations (3.13) and (3.15), and using estimates (3.14) and (3.16), we arrive at

$$
\frac{d}{d t}\left(|u|^{2}+|\omega|^{2}\right)+k_{1}\left(\|u\|^{2}+\|\left.\omega\right|^{2}\right) \leq \frac{1}{k_{1}}\left(\|f\|_{\mathbb{H}^{-1}}^{2}+\|g\|_{H^{-1}}^{2}\right) .
$$

Let us notice that (3.17) looks similar to (3.6). Therefore, proceeding in the same way we obtain

$$
\frac{1}{T} \int_{t}^{t+T}\left(\|u(s)\|^{2}+\|\omega(s)\|^{2}\right) d s \leq \frac{2}{k_{1}^{2}} \widetilde{F}_{-1}^{2}
$$

for $t$ and $T$ large enough.

Let us summarize the above results in the following lemma.

Lemma 1. Let $u_{0} \in H, \omega_{0} \in \dot{H}_{\text {per }}^{0}$ and $f \in L^{2}(0, T ; H) \cap L^{\infty}(0, T ; H), g \in$ $L^{2}\left(0, T, \dot{H}_{\mathrm{per}}^{0}\right) \cap L^{\infty}\left(0, T, \dot{H}_{\mathrm{per}}^{0}\right)$ for every $T>0$. Let $(u(t), \omega(t))$ be a solution of equations (1.1)-(1.3) with periodic boundary condition (1.5). Then the following inequalities hold for $t$ and $T$ large enough: 


$$
\begin{aligned}
& |u(t)|^{2}+|\omega(t)|^{2} \leq \frac{2}{k_{2}^{2}} \widetilde{F}^{2}, \\
& \|u(t)\|^{2}+\|\omega(t)\|^{2} \leq \widehat{c}_{1} \widetilde{F}^{2} \exp \left(\widehat{c}_{2}+\widehat{c}_{3} \widetilde{F}^{4}\right), \\
& \frac{1}{T} \int_{t}^{t+T}\left(\|u(s)\|^{2}+\|\omega(s)\|^{2}\right) d s \leq \frac{2}{k_{1} k_{2}} \widetilde{F}^{2}, \\
& \frac{1}{T} \int_{t}^{t+T}\left(\|u(s)\|^{2}+\|\omega(s)\|^{2}\right) d s \leq \frac{2}{k_{1}^{2}} \widetilde{F}_{-1}^{2}, \\
& \frac{1}{T} \int_{t}^{t+T}\left(|A u(s)|^{2}+|A \omega(s)|^{2}\right) d s \\
& \quad \leq\left(\frac{5}{k_{1}^{2}}+\frac{32 \nu_{r}^{2}}{\alpha k_{1}^{2} k_{2}}\right) \widetilde{F}^{2}+\frac{16 C \widehat{c}_{1}}{\alpha^{2} \nu k_{1}^{2} k_{2}^{3}} \widetilde{F}^{6} \exp \left(\widehat{c}_{2}+\widehat{c}_{3} \widetilde{F}^{4}\right),
\end{aligned}
$$

where the constants $\widehat{c}_{1}, \widehat{c}_{2}, \widehat{c}_{3}$ are defined in $(3.10), k_{1}=\min (\nu, \alpha), k_{2}=k_{1} \lambda_{1}$, and $\widetilde{F}$ and $\widetilde{F}_{-1}$ are defined in (3.1).

4. Determining modes. In this section we prove Theorem 2. We follow the method described in [5]. The proof is based on the following generalization of the classical Gronwall lemma.

Lemma 2. Let $\gamma=\gamma(t)$ and $\beta=\beta(t)$ be locally integrable real-valued functions on $\left[t_{0}, \infty\right)$ that satisfy the following conditions for some $T>0$ :

$$
\begin{aligned}
& \liminf _{t \rightarrow \infty} \frac{1}{T} \int_{t}^{t+T} \gamma(\tau) d \tau>0, \quad \limsup _{t \rightarrow \infty} \frac{1}{T} \int_{t}^{t+T} \gamma^{-}(\tau) d \tau<\infty, \\
& \lim _{t \rightarrow \infty} \frac{1}{T} \int_{t}^{t+T} \beta^{+}(\tau) d \tau=0,
\end{aligned}
$$

where $\gamma^{-}(t)=\max \{-\gamma(t), 0\}$ and $\beta^{+}(t)=\max \{\beta(t), 0\}$. Suppose that $\xi=$ $\xi(t)$ is an absolutely continuous nonnegative function on $\left[t_{0}, \infty\right)$ that satisfies the following inequality almost everywhere on $\left[t_{0}, \infty\right)$ :

$$
\frac{d \xi}{d t}+\gamma \xi \leq \beta
$$

Then $\xi(t) \rightarrow 0$ as $t \rightarrow \infty$.

Proof of Theorem 2. Writing the equations of the micropolar fluid in a functional form for a pair of solutions $\left(u_{1}, \omega_{1}\right)$ and $\left(u_{2}, \omega_{2}\right)$ and subtracting them we find

$$
\begin{aligned}
u_{t}+\left(\nu+\nu_{r}\right) A u+B\left(u, u_{1}\right)+B\left(u_{2}, u\right) & =2 \nu_{r} \operatorname{rot} \omega+f, \\
\omega_{t}+\alpha A_{1} \omega+B_{1}\left(u, \omega_{1}\right)+B_{1}\left(u_{2}, \omega\right)+4 \nu_{r} \omega & =2 \nu_{r} \operatorname{rot} u+g,
\end{aligned}
$$


where $u=u_{1}-u_{2}, \omega=\omega_{1}-\omega_{2}, f=f_{1}-f_{2}$ and $g=g_{1}-g_{2}$. First we deal with equation (4.2). Multiplying it by $Q_{m} u$ and integrating over $Q$ we obtain

$$
\begin{aligned}
\frac{1}{2} \frac{d}{d t}\left|Q_{m} u\right|^{2}+\left(\nu+\nu_{r}\right)\left\|Q_{m} u\right\|^{2}+b\left(u, u_{1}, Q_{m} u\right)+b\left(u_{2}, u, Q_{m} u\right) \\
=2 \nu_{r}\left(\operatorname{rot} \omega, Q_{m} u\right)+\left(f, Q_{m} u\right) .
\end{aligned}
$$

We estimate the linear terms of the RHS of (4.4) as follows:

$$
\begin{aligned}
&\left(f, Q_{m} u\right) \leq\|f\|_{\mathbb{H}^{-1}}\left\|Q_{m} u\right\|_{\mathbb{H}^{1}} \leq \frac{\nu+2 \nu_{r}}{4}\left\|Q_{m} u\right\|^{2}+\frac{1}{\nu+2 \nu_{r}}\|f\|_{\mathbb{H}^{-1}}^{2}, \\
& 2 \nu_{r}\left(\operatorname{rot} \omega, Q_{m} u\right)=2 \nu_{r}\left[\left(P_{m} \operatorname{rot} \omega, Q_{m} u\right)+\left(Q_{m} \operatorname{rot} \omega, Q_{m} u\right)\right] \\
& \leq 2 \nu_{r}\left|Q_{m} \operatorname{rot} \omega\right| \cdot\left|Q_{m} u\right| \\
& \leq \frac{\alpha}{8}\left\|Q_{m}^{1} \omega\right\|^{2}+\frac{8 \nu_{r}^{2}}{\alpha}\left|Q_{m} u\right|^{2}
\end{aligned}
$$

because $\left(P_{m} \operatorname{rot} \omega, Q_{m} u\right)=0$. In order to estimate the form $b$ we write

$$
b\left(u, u_{1}, Q_{m} u\right)=b\left(P_{m} u, u_{1}, Q_{m} u\right)+b\left(Q_{m} u, u_{1}, Q_{m} u\right)
$$

and

$$
b\left(u_{2}, u, Q_{m} u\right)=b\left(u_{2}, P_{m} u, Q_{m} u\right),
$$

because $b(u, v, v)=0$. Using $(2.2),(2.4)$ and the Young inequality we infer that

$$
\begin{aligned}
& b\left(P_{m} u, u_{1}, Q_{m} u\right) \leq c_{1}\left|P_{m} u\right|^{1 / 2}\left\|P_{m} u\right\|^{1 / 2}\left|u_{1}\right|^{1 / 2}\left\|u_{1}\right\|^{1 / 2}\left\|Q_{m} u\right\|, \\
& b\left(Q_{m} u, u_{1}, Q_{m} u\right) \leq \frac{\nu+\nu_{r}}{8}\left\|Q_{m} u\right\|^{2}+\frac{2 c_{1}^{2}}{\nu+\nu_{r}}\left|Q_{m} u\right|^{2}\left\|u_{1}\right\|^{2}, \\
& b\left(u_{2}, P_{m} u, Q_{m} u\right) \leq c_{1}\left|u_{2}\right|^{1 / 2}\left\|u_{2}\right\|^{1 / 2}\left|P_{m} u\right|^{1 / 2}\left\|P_{m} u\right\|^{1 / 2}\left\|Q_{m} u\right\| .
\end{aligned}
$$

Now we treat equation (4.3) in a similar manner. Taking the scalar product with $Q_{m}^{1} \omega$ in $\dot{H}_{\text {per }}^{0}$ we obtain

$$
\begin{aligned}
\frac{1}{2} \frac{d}{d t}\left|Q_{m}^{1} \omega\right|^{2}+\alpha\left\|Q_{m}^{1} \omega\right\|^{2}+b_{1}\left(u, \omega_{1},\right. & \left.Q_{m}^{1} \omega\right)+b_{1}\left(u_{2}, \omega, Q_{m}^{1} \omega\right) \\
& =2 \nu_{r}\left(\operatorname{rot} u, Q_{m}^{1} \omega\right)+\left(g, Q_{m}^{1} \omega\right) .
\end{aligned}
$$

The terms of the RHS of (4.8) are estimated as follows:

$$
\begin{aligned}
\left(g, Q_{m}^{1} \omega\right) & \leq \frac{\alpha}{4}\left\|Q_{m}^{1} \omega\right\|^{2}+\frac{1}{\alpha}\|g\|_{H^{-1}}^{2}, \\
2 \nu_{r}\left(\operatorname{rot} u, Q_{m}^{1} \omega\right) & =2 \nu_{r}\left(Q_{m}^{1} \operatorname{rot} u, Q_{m}^{1} \omega\right) \leq \frac{\nu_{r}}{4}\left\|Q_{m} u\right\|^{2}+4 \nu_{r}\left|Q_{m}^{1} \omega\right|^{2},
\end{aligned}
$$


and the form $b_{1}$ by using $(2.2),(2.6)$ and Young's inequality:

$$
\begin{aligned}
b_{1}\left(P_{m} u, \omega_{1}, Q_{m}^{1} \omega\right) \leq & c_{1}\left|P_{m} u\right|^{1 / 2}\left\|P_{m} u\right\|^{1 / 2}\left|\omega_{1}\right|^{1 / 2}\left\|\omega_{1}\right\|^{1 / 2}\left\|Q_{m}^{1} \omega\right\|, \\
b_{1}\left(Q_{m} u, \omega_{1}, Q_{m}^{1} \omega\right) \leq & \frac{\nu+\nu_{r}}{8}\left\|Q_{m} u\right\|^{2}+\frac{c_{1}^{2}\left\|\omega_{1}\right\|^{2}}{2\left(\nu+\nu_{r}\right)}\left|Q_{m} u\right|^{2} \\
& +\frac{\alpha}{8}\left\|Q_{m}^{1} \omega\right\|^{2}+\frac{c_{1}^{2}\left\|\omega_{1}\right\|^{2}}{2 \alpha}\left|Q_{m}^{1} \omega\right|^{2}, \\
b_{1}\left(u_{2}, P_{m}^{1} \omega, Q_{m}^{1} \omega\right) \leq & c_{1}\left|u_{2}\right|^{1 / 2}\left\|u_{2}\right\|^{1 / 2}\left\|Q_{m}^{1} \omega\right\| \cdot\left|P_{m}^{1} \omega\right|^{1 / 2}\left\|P_{m}^{1} \omega\right\|^{1 / 2} .
\end{aligned}
$$

Adding (4.4) and (4.8) and using the foregoing estimates we arrive at

$$
\begin{aligned}
& \frac{d}{d t}\left(\left|Q_{m} u\right|^{2}+\left|Q_{m}^{1} \omega\right|^{2}\right)+k_{1}\left(\left\|Q_{m} u\right\|^{2}+\left\|Q_{m}^{1} \omega\right\|^{2}\right) \\
& \quad-\left(\left|Q_{m} u\right|^{2}+\left|Q_{m}^{1} \omega\right|^{2}\right)\left(\frac{16 \nu_{r}^{2}}{\alpha}+\frac{4 c_{1}^{2}}{k_{3}}\left(\left\|u_{1}\right\|^{2}+\left\|\omega_{1}\right\|^{2}\right)\right) \leq \beta(t)
\end{aligned}
$$

where $\beta(t)=$ all terms converging to 0 as $t \rightarrow \infty$, and $k_{3}=\min \left(\nu+\nu_{r}, \alpha\right)$. We make use of the inequalities $\lambda_{m+1}\left|Q_{m} u\right|^{2} \leq\left\|Q_{m} u\right\|^{2}$ and $\lambda_{m+1}\left|Q_{m}^{1} \omega\right|^{2} \leq$ $\left\|Q_{m}^{1} \omega\right\|^{2}$ in order to write (4.10) in a form which allows us to use the generalized Gronwall lemma (Lemma 2):

$$
\begin{aligned}
\frac{d}{d t}\left(\left|Q_{m} u\right|^{2}+\mid\right. & \left.\left.Q_{m}^{1} \omega\right|^{2}\right)+\left(\left|Q_{m} u\right|^{2}+\left|Q_{m}^{1} \omega\right|^{2}\right) \\
& \cdot\left(k_{1} \lambda_{m+1}-\frac{4 c_{1}^{2}}{k_{3}}\left(\left\|u_{1}\right\|^{2}+\left\|\omega_{1}\right\|^{2}\right)-\frac{16 \nu_{r}^{2}}{\alpha}\right) \leq \beta .
\end{aligned}
$$

Setting

$$
\xi(t)=\left|Q_{m} u\right|^{2}+\left|Q_{m}^{1} \omega\right|^{2}, \quad \gamma(t)=k_{1} \lambda_{m+1}-\frac{4 c_{1}^{2}}{k_{3}}\left(\left\|u_{1}\right\|^{2}+\left\|\omega_{1}\right\|^{2}\right)-\frac{16 \nu_{r}^{2}}{\alpha},
$$

we can write (4.11) in the form

$$
\frac{d \xi}{d t}+\gamma \xi \leq \beta
$$

Now we only have to check the assumptions of Lemma 2. In Lemma 1 we have shown that

$$
\frac{1}{T} \int_{t}^{t+T}\left(\left\|u_{1}(s)\right\|^{2}+\left\|\omega_{1}(s)\right\|^{2}\right) d s \leq \frac{2}{k_{1}^{2}} \widetilde{F}_{-1}^{2}
$$

To check the first condition in (4.1) we write

$$
\begin{aligned}
\liminf _{t \rightarrow \infty} \frac{1}{T} \int_{t}^{t+T} \gamma(s) d s & \geq k_{1} \lambda_{m+1}-\frac{16 \nu_{r}^{2}}{\alpha}-\limsup _{t \rightarrow \infty} \frac{2 c_{1}^{2}}{k_{3}}\left(\left\|u_{1}\right\|^{2}+\left\|\omega_{1}\right\|^{2}\right) \\
& \geq k_{1} \lambda_{m+1}-\frac{16 \nu_{r}^{2}}{\alpha}-\frac{8 c_{1}^{2}}{k_{1}^{2} k_{3}} \widetilde{F}_{-1}^{2}>0
\end{aligned}
$$


This assumption is satisfied for

$$
m \geq \frac{16 \nu_{r}^{2}}{d \lambda_{1} \alpha k_{1}}+\frac{8 c_{1}^{2}}{d \lambda_{1} k_{3} k_{1}^{3}} \widetilde{F}_{-1}^{2}
$$

because $\lambda_{m} \sim d m$. It is easy to check that if $m$ satisfies (4.12) then the second assumption in (4.1) also holds. The third condition is satisfied because $\beta(t) \rightarrow 0$ as $t \rightarrow \infty$. That ends the proof.

This estimate is similar to that obtained in [23] but in the present paper we have relaxed the convergence of forces and moments to be only in $H^{-1}$. Moreover, the estimate obtained is in terms of their $H^{-1}$ norm. The same reasoning works in the case of no-slip boundary conditions.

Corollaries. This part of the paper was inspired by the paper of J. C. Robinson [22], in which he showed how the distribution of a force through modes influences the dimension of a global attractor of the Navier-Stokes equation.

Suppose that the asymptotic strength of the forces and moments is equal to $\widetilde{F}$. We check how their spatial distribution influences the number of determining modes. We consider several cases and write down the calculations only for $f$ because calculations for $g$ are exactly the same.

1. Assume that the forces and moments act only in two scales and the norms of both modes are equal, i.e.

$$
\left|f_{n}(t)\right|^{2}=\left|f_{N}(t)\right|^{2}=|f(t)|^{2} / 2
$$

Then the $\mathbb{H}^{-1}$ norm of $f$ satisfies

$$
\|f\|_{\mathbb{H}^{-1}}^{2}=\frac{|f|^{2}}{2}\left(\frac{1}{\lambda_{n}}+\frac{1}{\lambda_{N}}\right) \sim|f|^{2}\left(\frac{1}{n}+\frac{1}{N}\right) .
$$

Inserted into (4.12), this gives

$$
m \geq \frac{16 \nu_{r}^{2}}{d \lambda_{1} \alpha k_{1}}+\frac{8 c_{1}^{2}}{d \lambda_{1} k_{3} k_{1}^{3}} \widetilde{F}^{2}\left(\frac{1}{n}+\frac{1}{N}\right) .
$$

The number of determining modes depends on the inverse of the number of modes in which the forces and moments are acting.

2. Suppose that the forces and moments act only in some scales and the energy in each mode is not known,

$$
f=\sum_{k=n}^{N} f_{k} w_{k}
$$

The following inequalities are straightforward consequences of the definition of the norm in $\mathbb{H}^{-1}$ :

$$
\frac{1}{\lambda_{N}}|f|^{2} \leq\|f\|_{\mathbb{H}^{-1}}^{2} \leq \frac{1}{\lambda_{n}}|f|^{2}
$$


which implies

$$
\frac{1}{\lambda_{N}} \widetilde{F}^{2} \leq \widetilde{F}_{-1}^{2} \leq \frac{1}{\lambda_{n}} \widetilde{F}^{2}
$$

Inserting (4.13) to (4.12) we get

$$
m \geq \frac{16 \nu_{r}^{2}}{d \lambda_{1} \alpha k_{1}}+\frac{8 c_{1}^{2}}{d \lambda_{1} k_{3} k_{1}^{3}} \widetilde{F}_{-1}^{2} \geq \frac{16 \nu_{r}^{2}}{d \lambda_{1} \alpha k_{1}}+\frac{8 c_{1}^{2}}{d \lambda_{1} k_{3} k_{1}^{3}} \frac{\widetilde{F}^{2}}{\lambda_{N}} .
$$

3. Assume that the forces are uniformly distributed in the first $N$ modes, that is, $\left|f_{k}\right|^{2}=(1 / N)|f|_{L^{2}}^{2}$. Then

$$
\frac{\|f\|_{\mathbb{H}^{-1}}^{2}}{|f|^{2}}=\frac{1}{N} \sum_{k=1}^{N} \lambda_{k}^{-1}
$$

Since $\lambda_{k} \sim k$ we have $\widetilde{F}_{-1} \sim N^{-1 / 2}(\ln N)^{1 / 2} \widetilde{F}$, which yields the following estimate of the number of determining modes:

$$
m \geq \frac{16 \nu_{r}^{2}}{d \lambda_{1} k_{3} k_{1}}+\frac{8 c_{1}^{2}}{d \lambda_{1} k_{3} k_{1}^{3}} \widetilde{F}^{2} N^{-1 / 2}(\ln N)^{1 / 2} .
$$

4. Suppose that the forces and moments act in the first $N$ modes and the norm of a mode increases linearly with its number,

$$
\left|f_{k}\right|^{2}=\frac{2\|f\|_{L^{2}}^{2}}{N(N+1)} k
$$

for $k=1, \ldots, N$. Then

$$
\|f\|_{\mathbb{H}^{-1}}^{2}=\sum_{k=1}^{n} \frac{1}{\lambda_{k}} \frac{2 k\|f\|_{L^{2}}^{2}}{n(n+1)}=\frac{2\|f\|_{L^{2}}^{2}}{n(n+1)} \sum_{k=1}^{n} \frac{k}{\lambda_{k}} .
$$

Since $\lambda_{k} \sim k$ we have

$$
\|f\|_{\mathbb{H}^{-1}}^{2} \sim \frac{\|f\|_{L^{2}}^{2}}{n+1}
$$

and (4.12) implies

$$
m \geq \frac{16 \nu_{r}^{2}}{d \lambda_{1} k_{3} k_{1}}+\frac{8 c_{1}^{2}}{d \lambda_{1} k_{3} k_{1}^{3}} \frac{\widetilde{F}^{2}}{n+1} .
$$

5. Suppose again that the forces and moments act in the first $N$ modes, but now we assume that the norm of a mode decreases linearly with its number, that is,

$$
\left|f_{k}\right|^{2}=\frac{2\|f\|_{L^{2}}^{2}}{n(n+1)}(n+1-k), \quad k=1, \ldots, N
$$


Then

$$
\begin{aligned}
\|f\|_{H^{-1}}^{2} & =\frac{2\|f\|_{L^{2}}^{2}}{n(n+1)} \sum_{k=1}^{n} \frac{n+1-k}{\lambda_{k}} \approx \frac{2\|f\|_{L^{2}}^{2}}{n(n+1)} \sum_{k=1}^{n}\left(\frac{n+1}{k}-1\right) \\
& \approx \frac{2\|f\|_{L^{2}}^{2}}{n(n+1)}[(n+1) \ln n-n] .
\end{aligned}
$$

Inserting the above into (4.12) we infer that

$$
m \geq \frac{16 \nu_{r}^{2}}{d \lambda_{1} k_{3} k_{1}}+\frac{8 c_{1}^{2}}{d \lambda_{1} k_{3} k_{1}^{3}} \frac{2 \widetilde{F}^{2}}{n(n+1)}[(n+1) \ln n-n] .
$$

The above considerations show that if we increase the number of modes in which the forces and moments act, or we act only in modes with high wavenumber, then the number of modes necessary to determine the flow decreases. It could be so because in small scales, corresponding to highwavenumber modes, the damping effect of viscosity is stronger than in large scales. Moreover the number of determining modes depends on how the forces and moments are distributed throughout the modes.

The same argument can be applied to check how the distribution of the forces and moments influences the estimates of the number of determining nodes and the dimension of the global attractor.

5. Determining nodes. In this section we prove Theorem 3. We will actually show that (2.11), (2.14) and (2.15) imply that the solutions converge to each other in a stronger norm associated with enstrophy, that is,

$$
\int_{Q}\left(\left|\nabla u_{1}(x, t)-\nabla u_{2}(x, t)\right|^{2}+\left|\nabla \omega_{1}(x, t)-\nabla \omega_{2}(x, t)\right|^{2}\right) d x \rightarrow 0 \quad \text { as } t \rightarrow \infty .
$$

Set

$$
\eta(w)=\max _{1 \leq j \leq N}\left|w\left(x^{j}\right)\right|
$$

for each velocity or microrotation field $w$.

In the proof of existence of a finite set of determining nodes, two lemmas are used. One of them is the generalized Gronwall lemma, already applied in the previous section, the other is the following lemma from [12].

Lemma 3. Let the domain $Q$ be covered by $N$ identical squares $Q_{i}$. Consider the set $\Sigma=\left\{x^{1}, \ldots, x^{N}\right\}$ of points in $Q$, one in each square. Then, for each vector field $w$ in $\dot{\mathbb{H}}_{\text {per }}^{2}$,

$$
\begin{aligned}
|w|^{2} & \leq \frac{c}{\lambda_{1}} \eta(w)^{2}+\frac{c}{\lambda_{1}^{2} N^{2}}|\Delta w|^{2}, \\
\|w\|^{2} & \leq c N \eta(w)^{2}+\frac{c}{\lambda_{1} N}|\Delta w|^{2},
\end{aligned}
$$




$$
\|w\|_{L^{\infty}(Q)}^{2} \leq c N \eta(w)^{2}+\frac{c}{\lambda_{1} N}|\Delta w|^{2},
$$

for an appropriate constant $c$.

Proof of Theorem 3. Set $u=u_{1}-u_{2}, f=f_{1}-f_{2}$ etc. Subtracting the equations for $u_{1}$ and $u_{2}$ we find

$$
\frac{\partial u}{\partial t}+\left(\nu+\nu_{r}\right) \Delta u+\left(u_{1} \cdot \nabla\right) u_{1}-\left(u_{2} \cdot \nabla\right) u_{2}=2 \nu_{r} \operatorname{rot} \omega+f .
$$

By taking the inner product of (5.4) and $A u$ in $H$, we get

$$
\begin{aligned}
\frac{1}{2} \frac{d}{d t}\|u(t)\|^{2}+\left(\nu+\nu_{r}\right)|A u|^{2}+b\left(u, u_{1}, A u\right)+b\left(u_{2}, u, A u\right) \\
=2 \nu_{r}(\operatorname{rot} \omega, A u)+(f, A u) .
\end{aligned}
$$

Exploiting the orthogonality property (2.3) we obtain (cf. [5])

$$
b\left(u, u_{1}, A u\right)+b\left(u_{2}, u, A u\right)=-b\left(u, u, A u_{1}\right),
$$

thus we can write (5.5) in the form

(5.6) $\frac{1}{2} \frac{d}{d t}\|u(t)\|^{2}+\left(\nu+\nu_{r}\right)|A u|^{2}=2 \nu_{r}(\operatorname{rot} \omega, A u)+(f, A u)+b\left(u, u, A u_{1}\right)$.

We estimate the terms of the RHS of (5.6) using (2.5), Lemma 3 and Young's inequality:

$$
\begin{aligned}
2 \nu_{r}(\operatorname{rot} \omega, A u) \leq & \frac{\nu_{r}}{2}|A u|^{2}+2 \nu_{r}\|\omega\|^{2}, \\
(f, A u) \leq & \frac{\nu_{r}}{2}|A u|^{2}+\frac{1}{2 \nu_{r}}|f|^{2}, \\
b\left(u, u, A u_{1}\right) \leq & \frac{c^{1 / 4} c_{1}}{\lambda_{1}^{1 / 4}} \eta(u)^{1 / 2}\|u\| \cdot|A u|^{1 / 2}\left|A u_{1}\right| \\
& +\frac{c^{1 / 2} c_{1}^{2}}{\lambda_{1} N \nu}\|u\|^{2}\left|A u_{1}\right|^{2}+\frac{\nu}{4}|A u|^{2} .
\end{aligned}
$$

Using the above estimates we find from (5.6) that

$$
\begin{aligned}
\frac{1}{2} \frac{d}{d t}\|u\|^{2} & +\frac{3}{4} \nu|A u|^{2}-\frac{c^{1 / 2} c_{1}^{2}}{\lambda_{1} N \nu}\|u\|^{2}\left|A u_{1}\right|^{2} \\
& \leq 2 \nu_{r}\|\omega\|^{2}+\frac{1}{2 \nu_{r}}|f|^{2}+\frac{c^{1 / 4} c_{1}}{\lambda_{1}^{1 / 4}} \eta(u)^{1 / 2}\|u\| \cdot|A u|^{1 / 2}\left|A u_{1}\right| .
\end{aligned}
$$

Now we treat the equations for microrotation in a similar way. Subtracting them we find

$$
\frac{\partial \omega}{\partial t}+\alpha A_{1} \omega+\left(u_{1} \cdot \nabla\right) \omega_{1}-\left(u_{2} \cdot \nabla\right) \omega_{2}+4 \nu_{r} \omega=2 \nu_{r} \operatorname{rot} u+g .
$$


Multiplying (5.8) by $A_{1} \omega$ and integrating over $Q$ we get

$$
\begin{aligned}
\frac{1}{2} \frac{d}{d t}\|\omega\|^{2}+\alpha\left|A_{1} \omega\right|^{2} b_{1}\left(u, \omega, A_{1} \omega\right) & +b_{1}\left(u_{2}, \omega, A_{1} \omega\right)+4 \nu_{r}\|\omega\|^{2} \\
& =2 \nu_{r}\left(\operatorname{rot} u, A_{1} \omega\right)+\left(g, A_{1} \omega\right) .
\end{aligned}
$$

We estimate the nonlinear terms using (2.7), (2.8) and the Young inequality:

$$
\begin{aligned}
& b_{1}\left(u, \omega_{1}, A_{1} \omega\right) \leq \frac{\alpha}{8}\left|A_{1} \omega\right|^{2}+\frac{\nu}{4}|A u|^{2}+\frac{4 c_{1}^{4}}{\alpha^{2} \nu}|u|^{2}\left\|\omega_{1}\right\|^{4}, \\
& b_{1}\left(u_{2}, \omega, A_{1} \omega\right) \leq \frac{\alpha}{8}\left|A_{1} \omega\right|^{2}+\frac{2 c_{1}^{2}}{\alpha}\left|u_{2}\right| \cdot\left|A u_{2}\right| \cdot\|\omega\|^{2} .
\end{aligned}
$$

The terms of the RHS of (5.9) are estimated similar to the terms of the RHS of $(5.6)$ :

$$
\begin{aligned}
2 \nu_{r}\left(\operatorname{rot} u, A_{1} \omega\right) & \leq \frac{\alpha}{8}\left|A_{1} \omega\right|^{2}+\frac{8 \nu_{r}^{2}}{\alpha}\|u\|^{2}, \\
\left(g, A_{1} \omega\right) & \leq \frac{\alpha}{8}\left|A_{1} \omega\right|^{2}+\frac{2}{\alpha}|g|^{2} .
\end{aligned}
$$

Using the above estimates in (5.9) gives

$$
\begin{aligned}
\frac{1}{2} \frac{d}{d t}\|\omega\|^{2}+\frac{\alpha}{2}\left|A_{1} \omega\right|^{2}+4 \nu_{r}\|\omega\|^{2} & -\frac{8 \nu_{r}^{2}}{\alpha}\|u\|^{2}-\frac{2 c_{1}^{2}}{\alpha}\left|u_{2}\right| \cdot\left|A u_{2}\right| \cdot\|\omega\|^{2} \\
& \leq \frac{2}{\alpha}|g|^{2}+\frac{\nu}{4}|A u|^{2}+\frac{4 c_{1}^{4}}{\alpha^{2} \nu}|u|^{2}\left\|\omega_{1}\right\|^{4} .
\end{aligned}
$$

Adding (5.7) and (5.10) we obtain

$$
\begin{aligned}
\frac{1}{2} \frac{d}{d t}\left(\|u\|^{2}+\|\omega\|^{2}\right) & +\frac{k_{1}}{2}\left(|A u|^{2}+\left|A_{1} \omega\right|^{2}\right)-\|u\|^{2}\left(\frac{c_{1}^{2} c^{1 / 2}}{\lambda_{1} N \nu}\left|A u_{1}\right|^{2}+\frac{8 \nu_{r}^{2}}{\alpha}\right) \\
& -\|\omega\|^{2}\left(\frac{2 c_{1}}{\alpha}\left|u_{2}\right| \cdot\left|A u_{2}\right|-2 \nu_{r}\right)-\frac{4 c_{1}^{4}}{\alpha^{2} \nu}|u|^{2}\left\|\omega_{1}\right\|^{4} \\
\leq & \frac{2}{k_{3}}\left(|f|^{2}+|g|^{2}\right)+\frac{c_{1} c^{1 / 4}}{\lambda_{1}^{1 / 4}} \eta(u)^{1 / 2}\|u\| \cdot|A u|^{1 / 2}\left|A u_{1}\right|,
\end{aligned}
$$

where $k_{3}=\min \left\{\nu_{r}, \alpha\right\}$. From Lemma 3 we deduce

$$
|A u|^{2} \geq \frac{\lambda_{1} N}{c}\|u\|^{2}-\lambda_{1} N^{2} \eta(u)^{2},
$$

and an analogous inequality for $\omega$,

$$
\left|A_{1} \omega\right|^{2} \geq \frac{\lambda_{1} N}{c}\|\omega\|^{2}-\lambda_{1} N^{2} \eta(\omega)^{2} .
$$

By the Poincaré inequality we get

$$
\frac{4 c_{1}^{4}}{\alpha^{2} \nu}|u|^{2}\left\|\omega_{1}\right\|^{4} \leq \frac{4 c_{1}^{4}}{\alpha^{2} \nu \lambda_{1}}\|u\|^{2}\left\|\omega_{1}\right\|^{4} .
$$


Taking into account (5.12)-(5.14) we infer from (5.11) that

$$
\begin{aligned}
\frac{1}{2} \frac{d}{d t}\left(\|u\|^{2}+\|\omega\|^{2}\right) & +\left(\frac{k_{1} \lambda_{1} N}{c}-\frac{c_{1}^{2} c^{1 / 2}}{\lambda_{1} N \nu}\left|A u_{1}\right|^{2}-\frac{8 \nu_{r}^{2}}{\alpha}-\frac{2 c_{1}}{\alpha}\left|u_{2}\right| \cdot\left|A u_{2}\right|\right. \\
& \left.+2 \nu_{r}-\frac{4 c_{1}^{4}}{\alpha^{2} \nu \lambda_{1}}\left\|\omega_{1}\right\|^{4}\right)\left(\|u\|^{2}+\|\omega\|^{2}\right) \\
\leq & \frac{2}{k_{3}}\left(|f|^{2}+|g|^{2}\right)+\frac{c_{1} c^{1 / 4}}{\lambda_{1}^{1 / 4}} \eta(u)^{1 / 2}\|u\| \cdot|A u|^{1 / 2}\left|A u_{1}\right| \\
& +\frac{k_{1} \lambda_{1} N^{2}}{2}\left(\eta(u)^{2}+\eta(\omega)^{2}\right) .
\end{aligned}
$$

Setting

$$
\begin{aligned}
\gamma(t)= & 2\left(\frac{k_{1} \lambda_{1} N}{c}-\frac{c_{1}^{2} c^{1 / 2}}{\lambda_{1} N \nu}\left|A u_{1}\right|^{2}-\frac{8 \nu_{r}^{2}}{\alpha}-\frac{2 c_{1}}{\alpha}\left|u_{2}\right| \cdot\left|A u_{2}\right|\right. \\
& \left.+2 \nu_{r}-\frac{4 c_{1}^{4}}{\alpha^{2} \nu \lambda_{1}}\left\|\omega_{1}\right\|^{4}\right) \\
\beta(t)= & 2\left(\frac{2}{k_{3}}\left(|f|^{2}+|g|^{2}\right)+\frac{c_{1} c^{1 / 4}}{\lambda_{1}^{1 / 4}} \eta(u)^{1 / 2}\|u\| \cdot|A u|^{1 / 2}\left|A u_{1}\right|\right. \\
& \left.+\frac{k_{1} \lambda_{1} N^{2}}{2}\left(\eta(u)^{2}+\eta(\omega)^{2}\right)\right), \\
\xi(t)= & \|u\|^{2}+\|\omega\|^{2}
\end{aligned}
$$

we can write $(5.15)$ in the form

$$
\frac{d \xi}{d t}+\gamma \xi \leq \beta
$$

The time average of $\beta$ goes to zero as time goes to infinity because for $t$ bounded away from zero the time averages of the squares of the norms of $u_{1}$ and $u_{2}$ in $D(A)$ are uniformly bounded (Lemma 1). Therefore the third condition in (4.1) is satisfied. In order to check the first condition in (4.1) we write

$$
\begin{aligned}
\liminf _{t \rightarrow \infty} \frac{1}{T} \int_{t}^{t+T} \gamma(s) d s \geq & 2\left(\frac{k_{1} \lambda_{1} N}{c}+2 \nu_{r}-\frac{8 \nu_{r}^{2}}{\alpha}\right. \\
& -\limsup _{t \rightarrow \infty} \frac{1}{T} \int_{t}^{t+T}\left\{\frac{c_{1}^{2} c^{1 / 2}}{\lambda_{1} N \nu}\left|A u_{1}(s)\right|^{2}\right. \\
& \left.\left.+\frac{2 c_{1}}{\alpha}\left|u_{2}(s)\right| \cdot\left|A u_{2}(s)\right|+\frac{2 c_{1}^{4}}{\lambda_{1} \alpha \nu}\left\|\omega_{1}(s)\right\|^{4}\right\} d s\right)
\end{aligned}
$$




$$
\begin{aligned}
& \geq 2\left(\frac{k_{1} \lambda_{1} N}{c}+2 \nu_{r}-\frac{8 \nu_{r}^{2}}{\alpha}\right. \\
& \quad-\limsup _{t \rightarrow \infty} \frac{1}{T} \int_{t}^{t+T}\left\{\frac{c_{1}^{2} c^{1 / 2}}{\lambda_{1} N \nu}\left|A u_{1}(s)\right|^{2}+\frac{c_{1}}{\alpha}\left|u_{2}(s)\right|^{2}\right. \\
& \left.\left.\quad+\frac{c_{1}}{\alpha}\left|A u_{2}(s)\right|^{2}+\frac{4 c_{1}^{4}}{\lambda_{1} \alpha \nu}\left\|\omega_{1}(s)\right\|^{4}\right\} d s\right) \\
& \geq 2\left(\frac{k_{1} \lambda_{1} N}{c}+2 \nu_{r}-\frac{8 \nu_{r}^{2}}{\alpha}-\left(\frac{c_{1}^{2} c^{1 / 2}}{\lambda_{1} \nu}+\frac{c_{1}}{\alpha}\right)\right. \\
& \quad \cdot\left(\frac{5 \alpha k_{2}+32 \nu_{r}^{2}}{\alpha k_{1}^{2} k_{2}} \widetilde{F}^{2}+\frac{16 C \widehat{c}_{1}}{\alpha \nu k_{1}^{2} k_{2}^{3}} \widetilde{F}^{6} \exp \left(\widehat{c}_{2}+\widehat{c}_{3} \widetilde{F}^{4}\right)\right) \\
& \left.\quad-\frac{16 c_{1}^{4} \widehat{c}_{1}}{\lambda_{1} \alpha \nu k_{1} k_{2}} \widetilde{F}^{4} \exp \left(\widehat{c}_{2}+\widehat{c}_{3} \widetilde{F}^{4}\right)\right) .
\end{aligned}
$$

Therefore if

$$
\begin{aligned}
N \geq & \frac{c}{\lambda_{1} k_{1}}\left\{\frac{8 \nu_{r}^{2}}{\alpha}-2 \nu_{r}+\left(\frac{c_{1}^{2} c^{1 / 2}}{\lambda_{1} \nu}+\frac{c_{1}}{\alpha}\right)\right. \\
& \cdot\left(\frac{5 \alpha k_{2}+32 \nu_{r}^{2}}{\alpha k_{1}^{2} k_{2}} \widetilde{F}^{2}+\frac{16 C \widehat{c}_{1}}{\alpha \nu k_{1}^{2} k_{2}^{3}} \widetilde{F}^{6} \exp \left(\widehat{c}_{2}+\widehat{c}_{3} \widetilde{F}^{4}\right)\right) \\
& \left.+\frac{16 c_{1}^{4} \widehat{c}_{1}}{\lambda_{1} \alpha \nu k_{1} k_{2}} \widetilde{F}^{4} \exp \left(\widehat{c}_{2}+\widehat{c}_{3} \widetilde{F}^{4}\right)\right\},
\end{aligned}
$$

then the first condition in (4.1) is satisfied. It is easy to check that if $N$ satisfies (5.16) then the second condition in (4.1) also holds. Therefore we infer from the uniform Gronwall lemma that $\|u(t)\|^{2}+\|\omega(t)\|^{2} \rightarrow 0$ as $t \rightarrow \infty$. That ends the proof.

6. Hausdorff and fractal dimensions of the attractor. In this section we recall the notions of Hausdorff and fractal dimension of an invariant set. Then we prove Theorem 4, i.e. we show that the attractors $\mathcal{A}_{\nu_{r}}, \nu_{r} \geq 0$, associated with the system (1.1)-(1.3) have finite Hausdorff and fractal dimensions, which can be estimated by constants depending on the data: $f$, $g, \nu, \alpha$ and the domain $Q$ of the flow but independent of the microrotation viscosity $\nu_{r}$.

Let $(X, d)$ be a metric space and $Y \subset X$ be a subset of $X$. For every $d \in \mathbb{R}_{+}$and $\varepsilon>0$ we set

$$
\mu_{\mathrm{H}}(Y, d, \varepsilon)=\inf \sum_{i \in I} r_{i}^{d},
$$

where the infimum is taken over all coverings of $Y$ by a family $\left(B_{i}\right)_{i \in I}$ of 
balls of radii $r_{i} \leq \varepsilon \cdot \mu_{\mathrm{H}}(Y, d, \varepsilon)$ is a nonincreasing function of $\varepsilon$. The number $\mu_{\mathrm{H}}(Y, d)$, called the $d$-dimensional Hausdorff measure of $Y$, is defined as

$$
\mu_{\mathrm{H}}(Y, d)=\lim _{\varepsilon \rightarrow 0} \mu_{\mathrm{H}}(Y, d, \varepsilon)=\sup _{\varepsilon>0} \mu_{\mathrm{H}}(Y, d, \varepsilon) .
$$

If $\mu_{\mathrm{H}}\left(Y, d^{\prime}\right)<\infty$ for some $d^{\prime}$ then $\mu_{\mathrm{H}}(Y, d)=0$ for every $d>d^{\prime}$. Then there exists $d_{0} \in[0, \infty]$ such that $\mu_{\mathrm{H}}(Y, d)=0$ for every $d>d_{0}$ and $\mu_{\mathrm{H}}(Y, d)=\infty$ for $d<d_{0}$. The $d_{0}$ is called the Hausdorff dimension of $Y$, and denoted by $d_{\mathrm{H}}(Y)$.

Now we define the fractal dimension of $Y$. Let $\varepsilon>0$. We denote by $n_{Y}(\varepsilon)$ the minimum number of balls of $X$ of radius $\varepsilon$ necessary to cover $Y$. The fractal dimension of $Y$, also called the capacity of $Y$, is

$$
d_{F}(Y)=\limsup _{\varepsilon \rightarrow 0} \frac{\log n_{Y}(\varepsilon)}{\log 1 / \varepsilon} .
$$

We refer the reader to [4] for more details.

Proof of Theorem 4. The relation

$$
N-1<2 C_{0}\left(k_{1}^{3} k_{2}\right)^{-1 / 2}\left(|f|^{2}+|g|^{2}\right)^{1 / 2} \leq N
$$

is a consequence of estimates of the uniform Lyapunov exponents associated with the attractors $\mathcal{A}_{\nu_{r}}$.

First, we rewrite (1.1)-(1.3) in a more suitable form. To do this we introduce some notations. Let $\bar{u}_{i}=\left(u_{i}, \omega_{i}\right) \in \mathcal{H}$ (or $V$ ) for $i=1,2$. We introduce scalar products and norms in $\mathcal{H}$ and $\mathcal{V}$ as follows:

$$
\left[\bar{u}_{1}, \bar{u}_{2}\right]=\left(u_{1}, u_{2}\right)+\left(\omega_{1}, \omega_{2}\right), \quad[\bar{u}]=[\bar{u}, \bar{u}]^{1 / 2}
$$

for all $\bar{u}, \bar{u}_{1}, \bar{u}_{2} \in \mathcal{H}$, and

$$
\left[\left[\bar{u}_{1}, \bar{u}_{2}\right]\right]=\left(\nabla u_{1}, \nabla u_{2}\right)+\left(\nabla \omega_{1}, \nabla \omega_{2}\right), \quad[[\bar{u}]]=[[\bar{u}, \bar{u}]]^{1 / 2}
$$

for all $\bar{u}, \bar{u}_{1}, \bar{u}_{2} \in \mathcal{V}$. The notation seems to be confusing, but it will always be clear from context whether $(\cdot, \cdot)$ denotes the scalar product in $L^{2}$ or a vector in $\mathcal{H}$ or $\mathcal{V}$.

We define a trilinear form $B$ on $\mathcal{V} \times \mathcal{V} \times \mathcal{V}$ by

$$
B\left(\bar{u}_{1}, \bar{u}_{2}, \bar{u}_{3}\right)=b\left(u_{1}, u_{2}, u_{3}\right)+b_{1}\left(u_{1}, \omega_{2}, \omega_{3}\right)
$$

and associate with $B$ a bilinear continuous operator $\mathcal{B}$ from $\mathcal{V} \times \mathcal{V}$ to $\mathcal{V}^{\prime}$ as follows:

$$
\left\langle\mathcal{B}\left(\bar{u}_{1}, \bar{u}_{2}\right), \phi\right\rangle=B\left(\bar{u}_{1}, \bar{u}_{2}, \phi\right), \quad \bar{u}_{1}, \bar{u}_{2}, \phi \in \mathcal{V} .
$$

We define bilinear forms $R$ and $a$ on $\mathcal{V} \times \mathcal{V}$ by

$$
\begin{aligned}
R\left(\bar{u}_{1}, \bar{u}_{2}\right) & =-2 \nu_{r}\left(\operatorname{rot} \omega_{1}, u_{2}\right)-2 \nu_{r}\left(\operatorname{rot} u_{1}, \omega_{2}\right)+4 \nu_{r}\left(\omega_{1}, \omega_{2}\right), \\
a\left(\bar{u}_{1}, \bar{u}_{2}\right) & =\left(\nu+\nu_{r}\right)\left(\nabla u_{1}, \nabla u_{2}\right)+\alpha\left(\nabla \omega_{1}, \nabla \omega_{2}\right)
\end{aligned}
$$


and associate with them continuous linear operators $\mathcal{R}$ and $\mathcal{A}$ from $\mathcal{V}$ to $\mathcal{V}^{\prime}$ by

$$
\langle\mathcal{R}(\bar{u}), \phi\rangle=R(\bar{u}, \phi), \quad\langle\mathcal{A}(\bar{u}), \phi\rangle=a(\bar{u}, \phi), \quad \bar{u}, \phi \in \mathcal{V} .
$$

The weak form of equations (1.1)-(1.3) is (cf. [24])

$$
\begin{aligned}
\frac{d}{d t}(u(t), \varphi(t))+\left(\nu+\nu_{r}\right)(\nabla u(t), \nabla \varphi(t)) & +b(u(t), u(t), \varphi) \\
= & 2 \nu_{r}(\operatorname{rot} \omega(t), \varphi)+(f, \varphi)
\end{aligned}
$$

for all $\varphi \in V$, and

$$
\begin{aligned}
\frac{d}{d t}(\omega(t), \psi)+\alpha(\nabla \omega(t), \nabla \psi)+b_{1}(u(t) & , \omega(t), \psi)+4 \nu_{r}(\omega(t), \psi) \\
& =2 \nu_{r}(\operatorname{rot} u, \psi)+(g(t), \psi)
\end{aligned}
$$

for all $\psi \in \dot{H}_{\text {per }}^{1}(Q)$, in the sense of scalar distributions on $(0, \infty)$. Setting $G=(f, g) \in \mathcal{H}$ we can rewrite (6.2) and (6.3) as

$$
\frac{d}{d t}[\bar{u}, \phi]+a(\bar{u}, \phi)+B(\bar{u}, \bar{u}, \phi)+R(\bar{u}, \phi)=[G, \phi],
$$

where $\bar{u}=(u, \omega), \phi=(\varphi, \psi)$, or in the functional form

$$
\frac{d}{d t} \bar{u}+\mathcal{A}(\bar{u})+\mathcal{B}(\bar{u}, \bar{u})+\mathcal{R}(\bar{u})=G .
$$

The corresponding problem linearized about $\bar{u}$ has the form

$$
\frac{d}{d t} U=F^{\prime}(\bar{u}) U
$$

where $F^{\prime}(\bar{u})=-\mathcal{A}(U)-\mathcal{B}(\bar{u}, U)-\mathcal{B}(U, \bar{u})+\mathcal{R}(U)$.

Our aim is to estimate from above the trace

$$
\operatorname{Tr} F^{\prime}(\bar{u}) \circ \mathbb{P}_{N}=\sum_{i=1}^{N}\left[F^{\prime}(\bar{u}) \varphi_{j}, \varphi_{j}\right],
$$

where $\varphi_{j}=\varphi_{j}(\tau), j=1, \ldots, N$, is an orthonormal (in $\mathcal{H}$ ) basis of $\mathbb{P}_{N}(\tau) \mathcal{H}=$ $\operatorname{Span}\left\{U_{1}(\tau), \ldots, U_{N}(\tau)\right\}, \mathbb{P}_{N}\left(\tau, \xi_{1}, \ldots, \xi_{N}\right)$ being the orthogonal projector in $\mathcal{H}$ on the space spanned by $U_{j}(\tau), j=1, \ldots, N$, where the $U_{j}$ satisfy

$$
\frac{d}{d t} U_{j}=F^{\prime}(\bar{u}) U_{j}, \quad U_{j}(0)=\xi_{j}, \quad j=1, \ldots, N .
$$

Because $B\left(\bar{u}, \varphi_{j}, \varphi_{j}\right)=0$, we have

$$
\left[F^{\prime}(\bar{u}) \varphi_{j}, \varphi_{j}\right]=-a\left(\varphi_{j}, \varphi_{j}\right)-B\left(\varphi_{j}, \bar{u}, \varphi_{j}\right)-R\left(\varphi_{j}, \varphi_{j}\right) .
$$

Setting $\bar{u}=(u, \omega)$ and $\varphi_{j}=\left(v_{j}, z_{j}\right)$ we obtain 


$$
\begin{aligned}
-\sum_{i=1}^{N} a\left(\varphi_{j}, \varphi_{j}\right) & =-\left(\nu+\nu_{r}\right) \sum_{i=1}^{N}\left\|v_{j}\right\|^{2}-\alpha \sum_{i=1}^{N}\left\|z_{j}\right\|^{2}, \\
-\sum_{i=1}^{N} B\left(\varphi_{j}, \bar{u}, \varphi_{j}\right) & =-\sum_{i=1}^{N} b\left(v_{j}, u, v_{j}\right)-\sum_{i=1}^{N} b_{1}\left(v_{j}, \omega, z_{j}\right) \\
-\sum_{i=1}^{N} R\left(\varphi_{j}, \varphi_{j}\right) & =2 \nu_{r} \sum_{i=1}^{N}\left(\operatorname{rot} z_{j}, v_{j}\right)+2 \nu_{r} \sum_{i=1}^{N}\left(\operatorname{rot} v_{j}, z_{j}\right)-4 \nu_{r} \sum_{i=1}^{N}\left|z_{j}\right|^{2} .
\end{aligned}
$$

Now let us consider the operator $a+R$ :

$$
\begin{aligned}
a\left(\varphi_{j}, \varphi_{j}\right)+R\left(\varphi_{j}, \varphi_{j}\right)= & \left(\nu+\nu_{r}\right)\left(\left(v_{j}, v_{j}\right)\right)^{2}+\alpha\left(\left(z_{j}, z_{j}\right)\right)^{2} \\
& -2 \nu_{r}\left(\operatorname{rot} z_{j}, v_{j}\right)-2 \nu_{r}\left(\operatorname{rot} v_{j}, z_{j}\right)+4 \nu_{r}\left|z_{j}\right|^{2}
\end{aligned}
$$

By the first identity in (2.9), and Schwarz's and Young's inequalities, we have

$$
2 \nu_{r}\left(\operatorname{rot} z_{j}, v_{j}\right)+2 \nu_{r}\left(\operatorname{rot} v_{j}, z_{j}\right)=4 \nu_{r}\left(\operatorname{rot} z_{j}, v_{j}\right) \leq \nu_{r}\left\|v_{j}\right\|^{2}+4 \nu_{r}\left|z_{j}\right|^{2}
$$

therefore

$$
a\left(\varphi_{j}, \varphi_{j}\right)+R\left(\varphi_{j}, \varphi_{j}\right) \geq \nu\left(\left(v_{j}, v_{j}\right)\right)+\alpha\left(\left(z_{j}, z_{j}\right)\right) \geq k_{1}\left[\left[\varphi_{j}, \varphi_{j}\right]\right] \quad \forall \varphi_{j} \in V,
$$

where $k_{1}=\min \{\nu, \alpha\}$ as in (3.2), whence

$$
-\sum_{i=1}^{N}\left(a\left(\varphi_{j}, \varphi_{j}\right)+R\left(\varphi_{j}, \varphi_{j}\right)\right) \leq-k_{1} \sum_{i=1}^{N}\left[\left[\varphi_{j}, \varphi_{j}\right]\right]^{2} .
$$

We estimate the trilinear form $b$ as follows:

$$
\left|\sum_{i=1}^{N} b\left(v_{j}, u, v_{j}\right)\right|=\left|\sum_{i=1}^{N} \int_{Q}\left(v_{j} \cdot \nabla\right) u v_{j} d x\right| \leq \int_{Q}|\nabla u(x, t)| \varrho_{1}(x, t) d x
$$

where

$$
\varrho_{1}(x, t)=\sum_{i=1}^{N}\left|v_{j}(x, t)\right|^{2}
$$

and similarly

$$
\begin{aligned}
\left|\sum_{i=1}^{N} b_{1}\left(v_{j}, u, z_{j}\right)\right| & =\left|\sum_{i=1}^{N} \int_{Q}\left(v_{j} \cdot \nabla\right) u z_{j} d x\right| \\
& \leq \int_{Q}|\nabla u(x, t)| \varrho_{1}(x, t)^{1 / 2} \varrho_{2}(x, t)^{1 / 2} d x
\end{aligned}
$$

where

$$
\varrho_{1}(x, t)=\sum_{i=1}^{N}\left|z_{j}(x, t)\right|^{2}
$$


Therefore we can estimate the form $B$ as follows:

$$
\left|\sum_{i=1}^{N} B\left(\varphi_{j}, \bar{u}, \varphi_{j}\right)\right| \leq \int_{Q}\left(|\nabla u| \varrho_{1}+|\nabla \omega| \varrho_{1}^{1 / 2} \varrho_{2}^{1 / 2}\right) d x
$$

Setting $\varrho=\varrho_{1}+\varrho_{2}$, by the Cauchy and Schwarz inequalities we obtain

$$
\left|\sum_{i=1}^{N} B\left(\varphi_{j}, \bar{u}, \varphi_{j}\right)\right| \leq \int_{Q}(\varrho|\nabla u|+\varrho|\nabla \omega|) d x \leq \sqrt{2}|\varrho| \cdot[[\bar{u}]] .
$$

From the above estimates we infer that

$$
\operatorname{Tr} F^{\prime}(\bar{u}(\tau)) \circ \mathbb{P}_{N}(\tau) \leq-k_{1} \sum_{i=1}^{N}\left[\left[\varphi_{j}(\tau)\right]\right]^{2}+\sqrt{2}|\varrho(\tau)| \cdot[[\bar{u}(\tau)]] .
$$

Because the family $\left\{\varphi_{j}(\tau)\right\}_{j=1}^{N}$ is orthonormal in $\mathcal{H}$, the corresponding family of pairs $\left(v_{j}, z_{j}\right)$ is orthonormal in $L^{2}(Q)^{2 \times 2}$ and we can use a generalization of the Sobolev-Lieb-Thirring inequality ([26]) to write

$$
|\varrho(\tau)|^{2} \leq C_{0} \sum_{i=1}^{N}\left(\left\|v_{j}\right\|^{2}+\left\|z_{j}\right\|^{2}\right)=C_{0} \sum_{i=1}^{N}\left[\left[\varphi_{j}(\tau)\right]\right]^{2},
$$

for an appropriate constant $C_{0}$.

Since $\int_{Q} \varrho(x, t) d x=N$, by the Schwarz inequality we get

$$
N^{2} \leq|Q| \cdot|\varrho|^{2}
$$

and taking into account (6.5),

$$
\sum_{i=1}^{N}\left[\left[\varphi_{j}(\tau)\right]\right]^{2} \geq \frac{N^{2}}{C_{0}|Q|}
$$

We estimate the second term of the RHS of (6.4) using Young's inequality, (6.5) and (6.6):

$$
\sqrt{2}|\varrho| \cdot[[\bar{u}]] \leq \frac{k_{1}}{2 C_{0}}|\varrho|^{2}+\frac{C_{0}}{k_{1}}[[\bar{u}]]^{2} \leq \frac{k_{1}}{2} \sum_{i=1}^{N}\left[\left[\varphi_{j}\right]\right]^{2}+\frac{C_{0}}{k_{1}}[[\bar{u}]]^{2} .
$$

From the above considerations, we have

$$
\operatorname{Tr} F^{\prime}(\bar{u}(\tau)) \circ \mathbb{P}_{N}(\tau) \leq-\frac{k_{1}}{2} \frac{N^{2}}{C_{0}|Q|}+\frac{C_{0}}{k_{1}}[[\bar{u}]]^{2} .
$$

Let $\bar{u}_{0}=\left(u_{0}, \omega_{0}\right) \in \mathcal{A}$ and $\bar{u}(\tau)=S(\tau) \bar{u}_{0}$. Set

$$
\begin{aligned}
q_{N}(t)=\sup _{\bar{u}_{0} \in \mathcal{A}} \sup \left\{\frac{1}{t} \int_{0}^{t} \operatorname{Tr} F^{\prime}\left(S(\tau) \bar{u}_{0}\right) \circ \mathbb{P}_{N}(\tau) d \tau:\right. & \\
& \left.\xi_{i} \in H,\left[\xi_{i}\right] \leq 1, i=1, \ldots, N\right\},
\end{aligned}
$$




$$
q_{N}=\limsup _{t \rightarrow \infty} q_{N}(t) .
$$

In view of $(6.7)$, we conclude that

$$
q_{N} \leq-\frac{k_{1}}{2 C_{0}|Q|} N^{2}+\frac{C_{0}}{k_{1}} \gamma,
$$

where

$$
\gamma=\lim _{t \rightarrow \infty} \sup _{\bar{u} \in \mathcal{A}} \frac{1}{t} \int_{0}^{t}\left[\left[S(\tau) \bar{u}_{0}\right]\right]^{2} d \tau .
$$

We can estimate $\gamma$ in terms of the data using (3.6). Since

$$
\frac{1}{t} \int_{0}^{t}\left[\left[S(t) \bar{u}_{0}\right]\right]^{2} \leq \frac{1}{k_{1} k_{2}}[G]^{2}+\frac{1}{t} \frac{1}{k_{1}}\left[\bar{u}_{0}\right]^{2},
$$

it follows that

$$
\gamma \leq \frac{1}{k_{1} k_{2}}[G]^{2}
$$

and by (6.8),

$$
q_{N} \leq-\frac{k_{1}}{2 C_{0}|Q|} N^{2}+\frac{C_{0}}{k_{1}^{2} k_{2}}[G]^{2} .
$$

Setting

$$
\kappa_{1}=\frac{k_{1}}{2 C_{0}|Q|}, \quad \kappa_{2}=\frac{C_{0}}{k_{1}^{2} k_{2}}[G]^{2},
$$

we can write $(6.9)$ in the form

$$
q_{N} \leq-\kappa_{1} N^{2}+\kappa_{2}
$$

The general theory provided in [26] allows us to conclude that the uniform Lyapunov exponents $\mu_{j}$ associated with the attractor satisfy

$$
\mu_{1}+\cdots+\mu_{j} \leq-\kappa_{1} j^{2}+\kappa_{2}, \quad \forall j \in \mathbb{N},
$$

and for the $N$ satisfying (cf. Lemma VI, 2.2 in [26])

$$
N-1<\left(\frac{2 \kappa_{2}}{\kappa_{1}}\right)^{1 / 2} \leq N
$$

the assertion holds.

The resulting estimate of the dimension of the global attractor is similar to an analogous estimate for the micropolar fluid equations with no-slip boundary conditions (cf. [18]), but is of a higher order than the analogous estimate for the Navier-Stokes equation with periodic boundary conditions. 
7. Conclusions. The lack of the orthogonality property of the form $b_{1}$, which was mentioned in Section 2, causes that the estimates of the numbers of determining modes, nodes and the dimension of the global attractor are more involved than the corresponding estimates for the Navier-Stokes equation in the space-periodic case. The reason is the following: the estimate of the square of the norm of a solution in $\mathcal{V}$ and the average of the square of the norm in $D(A)$ for the Navier-Stokes equation, which are analogous to (3.9) and (3.12), are proportional to $F^{2}$ (cf. [5]), where

$$
F=\limsup _{t \rightarrow \infty}\left(\int_{Q}|f(x, t)|^{2} d x\right)^{1 / 2} .
$$

Estimates (3.9) and (3.12) are exponential with respect to $\widetilde{F}$, which implies an exponential estimate of the number of determining nodes. We cannot obtain linear dependence of the number of determining modes on $\widetilde{F}$ because the form $b_{1}$ does not have the orthogonality property (2.3).

We check how the estimates we obtained depend on $\nu_{r}$. The case when $\nu_{r}$ is small is particularly interesting because if $\nu_{r}=0$ the micropolar fluid system reduces to the Navier-Stokes system and the velocity field $u$ becomes independent of the microrotation field $\omega$ :

$$
\begin{gathered}
u_{t}-\nu \Delta u+(u \cdot \nabla) u+\nabla p=f, \\
\operatorname{div} u=0, \\
\omega_{t}-\alpha \Delta \omega+(u \cdot \nabla) \omega=g .
\end{gathered}
$$

1. The estimate of the number of determining modes

$$
m \approx c_{1} \widetilde{F}_{-1}^{2}+c_{2}
$$

(cf. (4.12)) is similar to that for the Navier-Stokes equation ([5]) and the micropolar fluid equations ([23]), both with Dirichlet boundary conditions. The coefficient $c_{1}$ does not depend on $\nu_{r}$ but if $\nu_{r} \rightarrow 0$, then $c_{2} \rightarrow 0$, so if $\nu_{r}=0$ our estimate agrees with that for the Navier-Stokes equation.

2. The estimate of the number of determining nodes

$$
m \approx P(\widetilde{F})+Q(\widetilde{F}) \exp (R(\widetilde{F}))
$$

(cf. (5.16)), where $P, Q$ and $R$ are polynomials, is similar to that for the Navier-Stokes equation with Dirichlet boundary conditions ([5]). If $\nu_{r} \rightarrow 0$, then the above estimate remains exponential with respect to $\widetilde{F}$. Our result is much more involved than an analogous result for the Navier-Stokes equation in [5], where it was shown that the dependence is linear.

3 . The estimate (6.1) of the dimension of the global attractor does not depend on $\nu_{r}$ and is similar to such an estimate for the Navier-Stokes equation with Dirichlet boundary condition ([5]) and for the micropolar fluid equations with Dirichlet boundary condition ([18]). 
Acknowledgments. This research was supported by a KBN grant for 2007. The author wishes to express his thanks to anonymous reviewers for their comments leading to improvements of this paper.

\section{References}

[1] S. Agmon, Lectures on Elliptic Boundary Value Problems, Elsevier, New York, 1965.

[2] M. Boukrouche and G. Łukaszewicz, Attractor dimension estimate for plane shear flow of micropolar fluid with free boundary, Math. Methods Appl. Sci. 28 (2005), 1673-1694.

[3] C. R. Doering and J. D. Gibbon, Applied Analysis of the Navier-Stokes Equations, Cambridge Univ. Press, Cambridge, 1995.

[4] K. Falconer, The Geometry of Fractal Sets, Cambridge Univ. Press, 1985.

[5] C. Foiaş, O. Manley, R. Rosa and R. Temam, Navier-Stokes Equations and Turbulence, Cambridge Univ. Press, 2001.

[6] C. Foias, O. P. Manley, R. Temam and Y. Treve, Asymptotic analysis of the NavierStokes equations, Phys. D 9 (1983), 157-188.

[7] C. Foiaş and E. Olson, Finite fractal dimension and Hölder-Lipschitz parametrization, Indiana Univ. Math. J. 45 (1996), 603-616.

[8] C. Foiaş et G. Prodi, Sur le comportement global des solutions non-stationnaires des équations de Navier-Stokes en dimension 2, Rend. Sem. Mat. Univ. Padova 39 (1967), 1-34.

[9] C. Foiaş and R. Temam, Determination of the solutions of the Navier-Stokes equations by a set of nodal values, Math. Comp. 43 (1984), 117-133.

[10] B. R. Hunt and V. Y. Kaloshin, Regularity of embeddings of infinite-dimensional fractal sets into finite-dimensional spaces, Nonlinearity 12 (1999), 1263-1275.

[11] A. A. Ilyin and E. S. Titi, Sharp estimates for the number of degrees of freedom for the damped-driven 2D Navier-Stokes equations, J. Nonlinear Sci., to appear.

[12] D. A. Jones and E. S. Titi, Upper bounds on the number of determining modes, nodes and volume elements for Navier-Stokes equations, Indiana Univ. Math. J. 42 (1993), 875-887.

[13] A. N. Kolmogorov, The local structure of turbulence in incompressible viscous fluid for very large Reynolds numbers, C. R. (Doklady) Acad. Sci. USSR (N.S.) 30 (1941), 301-305.

[14] - On degeneration of isotropic turbulence in an incompressible viscous liquid, ibid. 31 (1941), 538-540.

[15] R. H. Kraichnan, Inertial ranges in two-dimensional turbulence, Phys. Fluids 10 (1967), 1417-1423.

[16] O. A. Ladyzhenskaya, The Mathematical Theory of Viscous Incompressible Flow, rev. English ed., translated from the Russian by R. A. Silverman, Gordon and Breach, New York, 1963.

[17] L. Landau et E. Lifshitz, Mécanique des Fluides, Physique Théorique, tome 6, Éditions Mir, Moscow, 1971.

[18] G. Łukaszewicz, Long time behavior of $2 D$ micropolar fluid flows, Math. Comput. Modelling 34 (2001), 487-509.

[19] R. Mañé, On the dimension of the compact invariant sets of certain nonlinear maps, in: Lecture Notes in Math. 898, Springer, New York, 1981, 230-242. 
[20] P. B. Mucha and W. Sadowski, Long time behaviour of a flow in infinite pipe conforming to slip boundary conditions, Math. Methods Appl. Sci. 28 (2005), 1867-1880.

[21] J. C. Robinson, Infinite-Dimensional Dynamical Systems, Cambridge Univ. Press, 2001.

[22] —, Low dimensional attractors arise from forcing at small scales, Phys. D 181 (2003), 39-44.

[23] P. Szopa, Determining modes for 2-D micropolar fluid flows, Math. Comput. Modelling 42 (2005), 1079-1088.

[24] - On existence of solutions for 2-D micropolar fluid equations with periodic boundary conditions, Math. Methods Appl. Sci 30 (2007), 331-346.

[25] R. Temam, Navier-Stokes Equations and Nonlinear Functional Analysis, SIAM, Philadelphia, 1995.

[26] - Infinite Dimensional Dynamical Systems in Mechanics and Physics, 2nd ed., Springer, New York, 1997.

Institute of Applied Mathematics and Mechanics

University of Warsaw

Banacha 2

02-097 Warszawa, Poland

E-mail: szopa@duch.mimuw.edu.pl

Received on 31.5.2007;

revised version on 12.10.2007 
